AperTO - Archivio Istituzionale Open Access dell'Università di Torino

Resolving the taxonomy of emerging zoonotic pathogens in the Trichophyton benhamiae complex

This is a pre print version of the following article:

Original Citation:

Availability:

This version is available http://hdl.handle.net/2318/1769591

since 2021-01-27T15:58:40Z

Published version:

DOI:10.1007/s13225-020-00465-3

Terms of use:

Open Access

Anyone can freely access the full text of works made available as "Open Access". Works made available under a Creative Commons license can be used according to the terms and conditions of said license. Use of all other works requires consent of the right holder (author or publisher) if not exempted from copyright protection by the applicable law. 
Pre-print version of the article

Resolving the taxonomy of emerging zoonotic pathogens in the Trichophyton benhamiae complex

Čmoková, A.a,b, Kolařík, M.b, Dobiáš, R.c,d, Hoyer, L.L.e, Janouškovcová, H.f, Kano, R.g, Kuklová, I.h, Lysková, P.i, Machová, L.a,b, Maier, T.j, Mallátová, N.k, Man, M.I, Mencl, K.m, Nenoff, P.n, Peano, A.o, Prausová, H.p, Stubbe, D.q, Uhrlaß, S.n, Větrovský, T.r, Wiegand, C.s, Hubka, V.a,bEmail Author View Correspondence (jump link)

Fungal Diversity Volume 104, Issue 1, September 2020, Pages 333-387

https://link.springer.com/article/10.1007/s13225-020-00465-3

\section{Resolving the taxonomy of emerging zoonotic pathogens in the Trichophyton benhamiae complex}

Adéla Čmoková ${ }^{1,2}$, Miroslav Kolařík ${ }^{1,2}$, Radim Dobiášs ${ }^{3}$, Lois L. Hoyer ${ }^{4}$, Helena Janouškovcová ${ }^{5}$, Rui Kano $^{6}$, Ivana Kuklová ${ }^{7}$, Pavlína Lysková ${ }^{8}$, Thomas Maier ${ }^{9}$, Nad’a Mallátováa ${ }^{10}$, Matěj Man ${ }^{11}$, Karel Mencl $^{12}$, Pietro Nenoff ${ }^{13}$, Andrea Peano ${ }^{14}$, Hana Prausová ${ }^{15}$, Dirk Stubbe ${ }^{16}$, Silke Uhrla ${ }^{13}$, Tomáś Větrovský $^{2}$, Cornelia Wiegand ${ }^{17}$, Vit Hubka ${ }^{1,2}$

${ }^{1}$ Department of Botany, Faculty of Science, Charles University, Prague, Benátská 2, Prague 2, 128 01, Czech Republic

${ }^{2}$ Laboratory of Fungal Genetics and Metabolism, Institute of Microbiology, Czech Academy of Science, Vídeňská 1083, Prague 4, 142 20, Czech Republic

${ }^{3}$ Institute of Public Health, Partyzánské náměstí 7, Ostrava, 702 00, Czech Republic

${ }^{4}$ Department of Pathobiology, University of Illinois at Urbana-Champaign, 2001 South Lincoln Avenue, Urbana, IL, 61802, USA

${ }^{5}$ Biomedical Center, Faculty of Medicine in Pilsen, Charles University, Alej Svobody 1655/76, Pilsen, 323 00, Czech Republic

${ }^{6}$ Department of Veterinary Pathobiology, Nihon University College of Bioresource Sciences, 1866 Kameino, Fujisawa, Kanagawa, 252-0880, Japan

${ }^{7}$ General University Hospital in Prague, U Nemocnice 2, Prague 2, 12108, Czech Republic

${ }^{8}$ Institute of Health in Ústí nad Labem, Sokolovská 60, Prague 8, 186 00, Czech Republic 
${ }^{9}$ Microbiological Laboratory/R\&D Bioanalytics, Bruker Daltonik GmbH, Fahrenheitstraße 4, Bremen, 2 28359, Germany

$3{ }^{10}$ Laboratory of Medical Parasitology and Mycology, Central Laboratories, Hospital České Budějovice, 4 Boženy Němcové 585/54, České Budějovice, 370 01, Czech Republic

$5{ }^{11}$ Institute of Botany of the ASCR, Zámek 1, Průhonice, 252 43, Czech Republic

$6 \quad{ }^{12}$ Pardubice Regional Hospital, Kyjevská 44, Pardubice, 53203, Czech Republic

$7 \quad{ }^{13}$ Laboratory of medical microbiology, Mölbiser Hauptstraße 8, Rötha OT Mölbis, 04571, Germany

$8 \quad{ }^{14}$ Department of Animal Productions, Epidemiology and Ecology, University of Turin, Largo Paolo

9 Braccini 2, Grugliasco (TO), 10095, Italy

$10{ }^{15}$ Clinical Veterinary Laboratory Labvet, Bubenské nábřeží 306, Prague 7, 170 00, Czech Republic

${ }^{16}$ Mycology and Aerobiology, Scientific Institute of Public Health, Rue J. Wytsmanstraat 14, Brussels, B-1050 Belgium

*corresponding author e-mail: vit.hubka@ gmail.com; phone: +420 739663218

Running head: Emerging pathogens in the Trichophyton benhamiae complex

${ }^{17}$ Department of Dermatology, University Hospital Jena, Bachstraße 18, Jena, 07740, Germany

\section{Abstract}

Species of the Trichophyton benhamiae complex are predominantly zoophilic pathogens with a worldwide distribution. These pathogens have recently become important due to their epidemic spread in pets and pet owners. Considerable genetic and phenotypic variability has been revealed in these emerging pathogens, but the species limits and host spectra have not been clearly elucidated. In this study, we used an approach combining phylogenetic analysis based on four loci, population-genetic data, phenotypic and physiological analysis, mating type gene characterization and ecological data to resolve the taxonomy of these pathogens. This approach supported the inclusion of nine species in the complex, including three new species and one new variety. Trichophyton benhamiae var. luteum var. nov. ("yellow phenotype" strains) is currently a major cause of zoonotic tinea corporis and capitis in Europe (mostly transmitted from guinea pigs). This variety exhibits unique phenotypic and ecological characteristics compared to T. benhamiae var. benhamiae and is distinguishable by using microsatellite markers but not with the conventional DNA sequence markers used here. We demonstrated that isolates of the "white phenotype" do not form a monophyletic group and are segregated into T. benhamiae var. benhamiae (mostly from North America; dogs), T. europaeum sp. nov. (mostly from Europe; guinea pigs), and T. japonicum sp. nov. (the major cause of zoonotic infections in Japan but also found in Europe; rabbits and guinea pigs). The name T. africanum sp. nov. is proposed for the "African" race of $T$. benhamiae. The extinction of one mating type gene and adaptation to different hosts have played important roles in the evolution of pathogens from the $T$. benhamiae complex. A microsatellite typing 


\section{5}

6

7

scheme consisting of ten markers was developed for the purpose of the epidemiological surveillance of these emerging pathogens. MALDI-TOF MS was able to discriminate between the newly proposed species and varieties, suggesting that this method is useful for identification in clinical practice.

\section{INTRODUCTION}

Dermatophytes are a group of fungal pathogens that cause inflammatory and contagious skin diseases that are usually referred to as dermatophytoses, tinea or ringworm. These are among the most common diseases of warm-blooded animals, including humans, and their prevalence can reach dozens of percent in both human and animal populations (Havlickova et al. 2008, Seebacher et al. 2008, Cafarchia et al. 2010, Duarte et al. 2010, Agnetti et al. 2014, Ahdy et al. 2016, Kupsch et al. 2017). The treatment and prevention of these infections in humans, companion animals and pets require a considerable amount of funding every year (Kane and Summerbell 1997, Chermette et al. 2008, Bond 2010, Benedict et al. 2018, Shenoy and Jayaraman 2019).

The incidence of zoonotic dermatomycoses transmitted to humans from livestock decreased significantly in developed countries with the intensification of agriculture, introduction of preventive measures (e.g., vaccination in cattle) and advances in treatment options (Borman et al. 2007, Lund et al. 2014). In contrast, zoonotic infections transmitted from pets remain an important public health concern worldwide (Hubka et al. 2018c). Microsporum canis and Trichophyton mentagrophytes remain major agents of dermatophytosis in many domestic animals and cause a significant number of zoonotic dermatophytoses in humans (Hayette and Sacheli 2015). In addition to these well-known causal agents, several emerging zoonotic pathogens are increasingly reported in both humans and pets, and most of them belong to the Trichophyton benhamiae complex.

The Trichophyton benhamiae complex currently comprises six species: T. benhamiae, $T$. bullosum, T. concentricum, T. erinacei, T. eriotrephon and T. verrucosum (Lysková et al. 2015, de Hoog et al. 2017). These species are predominantly zoophilic, with the exception of anthropophilic $T$. concentricum, an agent of tinea imbricata in tropical regions (Bonifaz et al. 2004, Pihet et al. 2008, Bonifaz and Vazquez-Gonzalez 2011). Trichophyton verrucosum, a cause of dermatophytosis in cattle and other ruminants, is one of the best-known members of the complex. It has a worldwide distribution and causes economic losses in the food (negative impacts on milk and meat production), hide and skin industries (Chermette et al. 2008, Bond 2010). The incidence of infections in cattle has decreased in many regions in response to vaccination programmes or changes in agricultural systems, and the rate of infections in humans has decreased proportionally (Seebacher et al. 2008; Lund et al. 2014). By contrast, a lack of prophylaxis accounts for the high infection rates observed in countries such as Italy (Moretti et al. 2013). Trichophyton verrucosum grows slowly in culture and frequently produces only chlamydospores as its main microscopic characteristic. In this respect, it is superficially very similar to T. bullosum, which causes infections in donkeys and horses, but is much less common and is geographically restricted to the Middle East, Africa and Europe (Sitterle et al. 2012, Lysková et al. 
2015, Sabou et al. 2018). Scant data are available on the distribution of T. eriotrephon, which is only known from several poorly documented cases of dermatophytosis in humans and dogs (RezaeiMatehkolaei et al. 2013, Hubka et al. 2018c, Sabou et al. 2018). The remaining two zoophilic species, T. benhamiae and T. erinacei, are currently considered emerging pathogens, as their incidence as a cause of infections in pets and humans has increased significantly in the last decade (Hubka et al. 2018c).

A strikingly high incidence of zoonotic T. benhamiae (syn. Arthroderma benhamiae) infections, contracted mostly from guinea pigs, is currently reported in various European countries. Although this species was considered less clinically important in recent decades, it became one of the most common agents of zoonotic dermatophytoses after 2010 (Symoens et al. 2013, Nenoff et al. 2014, Uhrlaß et al. 2015, Hubka et al. 2018b, Sabou et al. 2018). It has been shown that the prevalence of the pathogen in guinea pig breeds and pet shops reaches up to $90 \%$ (Drouot et al. 2009, Kupsch et al. 2017, Overgaauw et al. 2017, Guillot et al. 2018, Bartosch et al. 2019). Infections occur more frequently in young guinea pigs and are usually asymptomatic. The presence of skin lesions with hair loss (mostly on the muzzle, forehead, ears and around eyes) is also reported in some individuals (Kraemer et al. 2012, Kraemer et al. 2013). When transmitted to the human host, the infections manifest most commonly as highly inflammatory tinea of glabrous skin and tinea capitis and less commonly as onychomycosis (Nenoff et al. 2014, Skořepová et al. 2014). The presence of asymptomatic infections in animal hosts contributes to the successful spread of the pathogen between animals kept in groups. Such asymptomatic infections also facilitate transmission to pet owners and the occurrence of small familial outbreaks or general infections among pet breeders, pet shop workers and others. In addition to guinea pigs, this pathogen has been reported in dogs, rabbits, cats, North American porcupines, various small rodents and foxes (Aho 1980, Fréalle et al. 2007, Takeda et al. 2012, Sieklucki et al. 2014, Hiruma et al. 2015, Ziółkowska et al. 2015, Needle et al. 2019).

Trichophyton benhamiae was originally described from several dog and human infections in North America (Ajello and Cheng 1967). The same authors induced a sexual state of the fungus and demonstrated its heterothallic nature by using in vitro mating experiments. In subsequent years, Takashio (1974) recognized two races among strains of $T$. benhamiae based on biological compatibility experiments: an "Americano-European" race and an "African" race of Arthroderma benhamiae (Takashio 1974). Furthermore, two phenotypically different groups among strains of the AmericanoEuropean race have recently been recognized by different authors and designated the "yellow phenotype" and "white phenotype" strains (Symoens et al. 2013, Nenoff et al. 2014, Hiruma et al. 2015, Brasch et al. 2016). The characterization of mating type genes showed that the MAT1-1-1 idiomorph was significantly prevalent among strains of the yellow phenotype, while MAT1-2-1 prevailed among strains of the white phenotype (Symoens et al. 2013). Similar observations of a lack of one MAT gene or significant bias towards one MAT idiomorph have been made in several other primary pathogenic 
dermatophytes, while the prevalence of both mating types in a balanced ratio is common in geophilic species (Metin and Heitman 2017, Kosanke et al. 2018).

It was demonstrated that the vast majority of European infections are caused by yellow phenotype strains that emerged relatively recently (Symoens et al. 2013, Hubka et al. 2014, Nenoff et al. 2014, Uhrlaß et al. 2015). The first documented cases of infections due to yellow phenotype strains were recorded between 2002 and 2008 in France and Switzerland (Contet-Audonneau and Leyer 2010, Charlent 2011, Khettar and Contet-Audonneau 2012, Symoens et al. 2013). The first cases in Germany and the Czech Republic were described shortly before 2010, and the pathogen became rapidly epidemic during the following years. Currently, T. benhamiae is the most important agent of dermatophytoses transmitted from animals in the Czech Republic and Germany (Hubka et al. 2014, Nenoff et al. 2014, Uhrlaß et al. 2015, Hubka et al. 2018b, Kupsch et al. 2020). The origin of yellow phenotype strains of T. benhamiae and the reason for the sudden increase in the incidence of human and animal infections in Europe after 2010 are unknown. As the breeding of guinea pigs has been popular in Europe for decades, the epidemic cannot be explained by a change in pet owner behaviour. Therefore, the spread of a new virulent and highly transmissible genotype/lineage was hypothesized (Čmoková 2015, Hubka et al. 2018c). The occurrence of $T$. benhamiae infections in non-European countries is generally poorly known except for individual reported cases. This is mostly due to insufficient surveillance and a lack of long-term epidemiological studies supported by molecular-based identification of dermatophytes.

In contrast to yellow phenotype strains, white phenotype strains have probably existed long term worldwide. Sporadic human and animal infections due to white phenotype strains were described from various European countries, Japan and the USA before the widespread dispersal of yellow phenotype strains in Europe (Ajello and Cheng 1967, Takashio 1974, Aho 1980, Hejtmánek and Hejtmánková 1989, Kano et al. 1998). In Japan, white phenotype strains were first reported in 1996 from an infected rabbit (Kano et al. 1998); human cases were reported in the following years (Nakamura et al. 2002), and the infections were summarized by Kimura et al. (2015). The increasing number of people breeding pets, together with the increasing import of animals to Japan, is considered a cause of the increased incidence in Japan (Hiruma et al 2015, Kimura et al 2015, Takeda et al 2012). Chronology of reports of white and yellow phenotype strains in various countries is summarized in Figure 1.

The aim of this study was to elucidate the species boundaries, host spectrum, and population structure of emerging pathogens in the Trichophyton benhamiae complex. We examined a large set of clinical isolates associated with human and animal infections that were mostly collected in European countries but also in the USA and Japan. We conducted DNA sequencing of four genetic loci, phylogenetic analyses, and analyses of morphology and physiology to examine whether the previously detected level of phenotypic and genetic variability reflects undescribed species diversity or a high level of infraspecific variability. The levels of recombination/clonality within species and populations, respectively, were estimated by calculating the index of association and determining the ratios between MAT locus idiomorphs. MALDI-TOF MS spectra were compared between species of the T. benhamiae 
complex to test the possibility of their differentiation in the clinical setting. A set of highly variable microsatellite markers were developed to analyse the population structure and relationships between strains with differences in their geographic origin, host spectrum and phenotype. The new taxonomic classification and microsatellite typing scheme proposed in this study will enable the monitoring of changes in the frequencies of individual species and genotypes. It will help to evaluate the results of preventive measures and interventions and is a basic prerequisite for the development of epidemiological studies.

\section{MATERIALS AND METHODS}

\section{Source of isolates}

More than three hundred strains isolated from human and animal patients with dermatophytosis caused by $T$. benhamiae complex species were obtained for this study from various clinical laboratories, hospitals and universities (Table S1): Laboratory for Medical Microbiology (Mölbis, Germany), College of Veterinary Medicine, University of Illinois at Urbana-Champaign (USA), The University of Tokyo (Japan), School of Veterinary Medicine, University of Turin (Italy), and various institutions in the Czech Republic (Institute of Public Health in Ostrava and Prague, General University Hospital in Prague, University Hospital in Pilsen, Hospital České Budějovice, Hospital in Pardubice and Labvet veterinary laboratory in Prague). This set of strains was further supplemented with isolates from culture collections, especially BCCM/IHEM Biomedical Fungi and Yeasts Collection (Brussels, Belgium) and CBS culture collection housed at the Westerdijk Institute (Utrecht, The Netherlands).

Selected isolates were deposited into the Culture Collection of Fungi (CCF), Department of Botany, Charles University, Prague, Czech Republic; herbarium specimens of newly described species were deposited into the herbarium of the Mycological Department, National Museum in Prague, Czech Republic (PRM).

\section{Molecular studies}

DNA was extracted from seven-day-old colonies using the ArchivePure DNA Yeast and Gram2+ Isolation Kit (5 PRIME Inc., Gaithersburg, Maryland) according to the manufacturer's instructions as updated by Hubka et al. (2015b). The quality of the extracted DNA was evaluated by NanoDrop 1000 Spectrophotometer.

The ITS rDNA region (ITS1-5.8S-ITS2 cluster) was amplified using the primer set SR6R and LR1 (White et al. 1990) or ITS1F and ITS4 (White et al. 1990, Gardes and Bruns 1993), partial gapdh gene encoding glyceraldehyde-3-phosphate dehydrogenase was amplified with primers GPDF and GPDR (Kawasaki et al. 2011), partial tubb gene encoding $\beta$-tubulin with primers Bt2a and Bt2b (Glass and Donaldson 1995), and tefl $\alpha$ gene encoding translation elongation factor $1-\alpha$ with primers EFDermF and EF-DermR (Mirhendi et al. 2015). All primer combinations are listed in Table S2. Reaction volume of $20 \mu \mathrm{L}$ contained $1 \mu \mathrm{L}$ (50 ng mL-1) of DNA, $0.3 \mu \mathrm{L}$ of both primers ( $25 \mathrm{pM} \mathrm{mL}-1), 0.2 \mu \mathrm{L}$ 
of My Taq Polymerase and $4 \mu \mathrm{L}$ of $5 \times$ My Taq PCR buffer (Bioline, London, UK). PCR conditions followed protocol described by Hubka et al. (2018a). PCR product purification followed protocol of Réblová et al. (2016). Automated sequencing was performed at Macrogen Sequencing Service (Amsterdam, The Netherlands) using both terminal primers. The DNA sequences obtained in this study were deposited into the GenBank database (www.ncbi.nlm.nih.gov) under the accession numbers listed in Table 1.

\section{Phylogenetic analysis}

Alignments of the ITS, gapdh, tubb and tefl $\alpha$ regions were performed using the FFT-NS-i option implemented with the MAFFT online service (Katoh et al. 2017). The alignments were trimmed, concatenated and then analysed using maximum likelihood (ML) and Bayesian inference (BI) methods. Suitable partitioning schemes and substitution models (Bayesian information criterion) for the analyses were selected using a greedy strategy implemented in PartitionFinder 2 (Lanfear et al. 2017) with settings allowing introns, exons, codon positions and segments of the ITS region to be independent datasets. The optimal partitioning schemes for each analysed dataset along with basic alignment characteristics are listed in Table S3. The ML trees were constructed with IQ-TREE version 1.4.4 (Nguyen et al. 2015) with nodal support determined by nonparametric bootstrapping (BS) with 1000 replicates. The trees were rooted with Trichophyton rubrum. Bayesian posterior probabilities (PP) were calculated using MrBayes 3.2.6 (Ronquist et al. 2012). Optimal partitioning scheme and substitution models were selected as described above and are listed in Table S3. The analysis ran for $10^{7}$ generations, two parallel runs with four chains each were used, every $1000^{\text {th }}$ tree was retained, and the first $25 \%$ of trees were discarded as burn-in. The convergence of the runs and effective sample sizes were checked in Tracer v1.6 (http://tree.bio.ed.ac.uk/software/tracer).

The modified complex indel coding (MCIC) algorithm implemented in SeqState version 1.25 (Müller 2005) was used to code gaps. The TCS network method (Clement et al. 2000) was used to generate haplotype networks implemented in the program PopART (Leigh and Bryant 2015).

\section{Development of microsatellite markers}

Microsatellite motifs were identified in the available genomic sequence of T. europaeum CBS 112371 = IHEM 20161 (http://www.broadinstitute.org/) using WebSat online software (Martins et al. 2009). The same program suggested optimal primers for the amplification of target loci. We selected di-, tri-, and tetranucleotide repeats based on the loci with the highest repeat numbers. Interrupted repeats as well as loci containing two or more repeat motifs within the fragments delimited by particular primer pairs were excluded. A pilot set of eight strains was used to evaluate microsatellite polymorphism for all candidate loci following the method of Schulke (2000). PCR conditions were as follows: one cycle at $95{ }^{\circ} \mathrm{C}$ for $1 \mathrm{~min} ; 27$ cycles at $95^{\circ} \mathrm{C}$ for $30 \mathrm{~s}, 55^{\circ} \mathrm{C}$ for $30 \mathrm{~s}, 72{ }^{\circ} \mathrm{C}$ for $45 \mathrm{~s}$, followed by eight cycles at $95{ }^{\circ} \mathrm{C}$ for $30 \mathrm{~s}, 53{ }^{\circ} \mathrm{C}$ for $30 \mathrm{~s}, 72{ }^{\circ} \mathrm{C}$ for $45 \mathrm{~s}$ and a final extension at $72{ }^{\circ} \mathrm{C}$ for $10 \mathrm{~min}$. A set of 24 
loci exhibiting the highest level of polymorphism was selected from the 160 tested loci. The PCR products were screened for the presence of undesirable polymorphisms in the microsatellite flanking regions and the presence of polymorphisms in the microsatellite regions by sequencing. Emphasis was also placed on the selection of loci that were approximately uniformly distributed across the genome. Primer-primer interactions were checked before assembling multiplexes using Multiple Primer Analyzer (http://www.thermoscientificbio.com/webtools/multipleprimer/). The forward primers of ten selected loci were tagged with fluorescent dye and arranged into a single multiplex panel (Table 2). The reaction volume of $5 \mu \mathrm{L}$ for PCR contained $50 \mathrm{ng}$ DNA, $0.5 \mu \mathrm{L}$ of the mixture of primers and $2.5 \mu \mathrm{L}$ of Multiplex PCR Master Mix (QIAGEN, Germany). The PCR conditions were chosen according to the manufacturer's recommendations. The PCR products (diluted in water 1:50) were mixed with $10 \mu \mathrm{L}$ of deionized formamide and $0.2 \mu \mathrm{L}$ of the GeneScan ${ }^{\mathrm{TM}} 600 \mathrm{LIZ}$ size standard and denatured for $5 \mathrm{~min}$ at $95^{\circ} \mathrm{C}$, followed by analysis on an ABI 3100 Avant Genetic Analyzer.

\section{Statistical analysis of microsatellite data}

The discriminatory power of these newly designed loci was calculated using Simpson's index of diversity as described previously (Hunter and Gaston 1988). A binary and allele data matrix was created using GeneMarker 1.51 software (SoftGenetics, LLC, State College, PA, USA) and used to estimate the similarities between individuals using Jaccard's similarity coefficient calculation in the program FAMD (Schlueter and Harris 2006). A neighbour-joining tree based on Jaccard's similarity coefficient matrix was constructed using the same software. Genetic distances were calculated from the same matrix and used for the construction of the NeighborNet network in the SplitsTree 4 program (Huson 1998).

A Bayesian model-based clustering algorithm with a clustering number $(K)=1-10$ was applied to the allele data matrix using the software STRUCTURE (Pritchard et al. 2000). Ten simulations were calculated at the www.bioportal.uio.no server (Lifeportal, University of Oslo) using the admixture model and $10^{6} \mathrm{MCMC}$ replicates; $5 \times 10^{8}$ replicates were discarded as burn-in. The no-admixture model and uncorrelated allele frequencies were chosen for the analysis. The optimal clustering number $(\mathrm{K})$ was estimated using $\Delta \mathrm{K}$ and similarity coefficients (Evanno et al. 2005), and both values were calculated using the script structure-sum (Ehrich 2006) in the R version 3.3.4 program (R_Core_Team 2016).

The genetic variability within and between clusters was analysed for ten variable loci via analysis of molecular variance (AMOVA) in the Arlequin program (Schneider et al. 2000). The degree of gene flow among clusters was estimated using a pairwise fixation index ( $\left.\mathrm{F}_{\mathrm{ST}}\right)$ and a coefficient of genetic differentiation $\left(\mathrm{G}_{\mathrm{ST}}\right.$ ) calculated in Arlequin (Schneider et al. 2000) and POPGENE (Yeh et al. 1999), respectively.

The degree of clonality or recombination within particular clusters was estimated by calculating the index of association $\left(I_{A}\right)$ in the program MultiLocus 1.3 (Agapow and Burt 2001), which is used for 
measuring the linkage disequilibrium between alleles and is useful in inferring the occurrence of cryptic recombination in putatively asexual populations (Burt et al. 1996). Random mating is suggested if no linkage is detected between the alleles of different loci (randomly distributed alleles); in that case $\mathrm{I}_{\mathrm{A}}$ is expected to be nearly zero or zero. We tested for significant deviation from 10000 random multilocus permutations of genotypes under a random mating model.

To measure within-population diversity, Nei’s genotype diversity (Dg) was calculated based on frequencies of genetically distinct individuals, and Nei's gene diversity (D) was calculated based on the frequencies of alleles at individual loci (Nei 1987, Kosman 2003). The effective number of genotypes $\left(\mathrm{G}_{\text {eff }}\right)$ (Parker Jr 1979) was calculated based on the number of equally abundant genotypes required to reflect the value of a diversity measure. It was calculated to obtain diversity values comparable beween the clusters. The degree of genetic divergence was investigated by rarity index of (DW index; frequency down-weighted marker values) (Schönswetter and Tribsch 2005). All mentioned population indexes $\left(\mathrm{Dg}, \mathrm{D}, \mathrm{DW}, \mathrm{G}_{\mathrm{eff}}\right)$ were calculated from the binary data matrix using script AFLPdat (Ehrich 2006) in R 3.0.2. Frequency histograms of pairwise differences between individuals were generated using the same program.

\section{MAT locus determination and mating experiments}

A partial sequence of the MAT1-1-1 gene encoding the alpha box domain was amplified with the primers MF1 and MF5, and a partial sequence of the MAT1-2-1 gene encoding the high mobility group (HMG) domain was amplified with the primers Ab_HMG_F and Ab_HMG_R or TmHMG3S and TmHMG3R (Kano et al. 2012, Symoens et al. 2013, Kosanke et al. 2018). The PCR volume of $10 \mu \mathrm{L}$ contained $25 \mathrm{ng}$ of DNA, $0.15 \mu \mathrm{L}$ of both primers $\left(25 \mathrm{pM} \mathrm{mL}^{-1}\right), 0.15 \mu \mathrm{L}$ of My Taq Polymerase and $2 \mu \mathrm{L}$ of buffer. The PCR conditions were described above. The PCR products were visualized in an electrophoretogram ( $1 \%$ agarose gel with $0.5 \mu \mathrm{g} \mathrm{mL} \mathrm{m}^{-1}$ ethidium bromide). Several PCR products of each MAT idiomorph were subjected to sequencing for the confirmation of product specificity.

\section{Phenotypic studies}

Macromorphology. The morphology of the colonies on malt extract agar at $25^{\circ} \mathrm{C}$ was documented in all strains. At least five strains from each species (if available) were subjected to a detailed analysis that involved macromorphology on MEA, potato dextrose agar (PDA, Himedia, Mumbai, India) and Sabouraud dextrose agar [SAB, Atlas (2010)] at 25, 30 and $37{ }^{\circ} \mathrm{C}(\mathrm{SAB})$. The macromorphology of the colonies was documented using an Olympus SZ61 or Canon EOS 500D binocular loupe (with Olympus Camedia C-5050 Zoom camera) or Canon EOS 500D. Colony colour determinations were made using the ISCC-NBS Centroid Colour Charts (Kelly 1964); http://tx4.us/nbs/nbs-1.htm.

Micromorphology was documented using an Olympus BX-51 microscope. Particular micromorphological characteristics were recorded at least 35 times for each isolate (at least five strains selected per species). The variance inflation factor (VIF) was assessed before performing the analysis 
of variance to test the correlation between variables. Statistical differences in particular phenotypic characteristics were tested with one-way analysis of variance (ANOVA) followed by Tukey's honestly significant difference (HSD) test in program R version 3.3.4 (R_Core_Team 2016).

\section{MALDI-TOF MS}

The cultivation of strains from the T. benhamiae clade (five strains from each species available) was performed in liquid cultivation medium for $22-24 \mathrm{~h}$. The selected strains were prepared according to Schrödl et al. (2012) and analysed "as a blinde" by matrix-assisted laser desorption/ionization time-offlight mass spectrometry (MALDI-TOF MS). In brief, for MALDI-TOF MS analysis, all samples were prepared using the liquid cultivation method and ethanol / formic acid extraction method. One milliliter of each over night culture was centrifuged for $2 \mathrm{~min}$ at about 10,000 g $(=980 \mathrm{~cm} / \mathrm{s} \mathrm{g}=980 \mathrm{~cm} / \mathrm{s} 22$. The supernatant was carefully removed and the fungal pellet was resuspended in $1 \mathrm{ml}$ water, mixed thoroughly, and centrifuged for further $5 \mathrm{~min}$ at $10000 \times \mathrm{g}$. After removing the supernatant the pellet was resuspended in a mixture of $300 \mu \mathrm{L}$ bidistilled water and $900 \mu \mathrm{L}$ absolute ethanol. After centrifugation, the fungal cells were dried shortly and mixed thoroughly with $50 \mu \mathrm{L}$ of $70 \%$ formic acid and $50 \mu \mathrm{L}$ pure acetonitrile, followed by centrifugation for $2 \mathrm{~min}$ at $10000 \times \mathrm{g}$. A volume of $1 \mu \mathrm{L}$ supernatant was placed onto a MALDI target plate (Bruker Daltonik GmbH, Germany) and allowed to dry at room temperature. Eight MALDI target positions per strain were prepared in parallel. Each sample position (including one Bruker Bacterial Trest Standard position) was overlaid with $1 \mu \mathrm{L}$ of matrix (HCCA portioned; Bruker Daltonik GmbH, Germany) and air dried at room temperature. MALDI-TOF MS measurement was conducted on a Microflex LT benchtop instrument operated by FlexControl software (Bruker Daltonik GmbH, Leipzig, Germany). Spectra were acquired in linear positive mode at a laser frequency of $200 \mathrm{~Hz}$ within a mass range from 2000 to $20000 \mathrm{Da}$ by using the standard flexControl and AutoX methods. For each sampled spot up to three sum spectra were accumulated resulting in 24 MALDI spectra per strain. Finally, five spectra were selected for better spectra handling and visualization.

\section{RESULTS}

\section{Phylogeny of the Trichophyton benhamiae complex}

We assessed 340 combined ITS, gapdh, tubb and tefl- $\alpha$ sequences from members of the T. benhamiae species complex (TBSC) in the phylogenetic analysis. The final alignment included 2371 characters, with 247 variable and 152 parsimony informative sites, and Trichophyton rubrum CBS 202.88 was used as the outgroup. The detailed alignment characteristics together with the partitioning schemes and substitution models are listed in Table S3. The isolation source and accession numbers for the DNA sequences are available in Table 1 and Table S1. The alignments were deposited in the Dryad Digital Repository: https://doi.org/XXXXXX. 
Members of the TBSC were resolved into three major monophyletic clades in the best scoring multiple-gene ML tree shown in Figure 2, (single-gene trees are shown in Figure S2-S5).

The $\boldsymbol{T}$. benhamiae clade contains anthropophilic T. concentricum $(\mathrm{n}=3)$ and the EuropeanAmerican race of $T$. benhamiae $(\mathrm{n}=318)$. The isolates of the European-American race do not form a monophyletic lineage and are paraphyletic with respect to $T$. concentricum. These strains are segregated into three major subclades: $T$. benhamiae s. str. and two newly proposed species, $T$. japonicum sp. nov. and T. europaeum sp. nov. Isolates of $T$. benhamiae s. str. originating mostly from Europe and North America, and they comprise both white and yellow phenotype strains. They form a monophyletic and fully supported (100\% bootstrap supports, bs/1.00 posterior probability, pp) subclade together with $T$. concentricum, which can be differentiated by only two unique substitutions in the ITS region and three in the tefl- $\alpha$ gene (the $t u b b$ and gapdh genes are identical).

Species from the T. benhamiae clade show a low level of intraspecific genetic variability. In total, there are only seven unique multilocus genotypes (MLST) among 318 isolates belonging to the T. benhamiae clade (Figure 3). Two MLST genotypes are present among T. benhamiae strains, represented by a single substitution in the tefl- $\alpha$ gene (Figure S4). Two MLST genotypes are present in T. japonicum, caused by a single substitution in the ITS1 region. Trichophyton japonicum can be differentiated from closely related T. europaeum by a single substitution in the ITS region and three conserved substitutions in the gapdh gene (Figure S2-S5). No intraspecific variability is detectable among the isolates of T. europaeum. The only exception is the isolate of "T. europaeum" IHEM 25139, which presents an abnormal ITS1 region sequence that contains 6 additional substitutions compared to the $T$. europaeum isolates. Some of these positions are critical for the differentiation of the $T$. europaeum/T. japonicum lineage from T. benhamiae s. str., suggesting that this strain could be hybrid between T. benhamiae clade species. The gapdh gene sequence of IHEM 25139 is typical of $T$. europaeum.

Both MAT gene idiomorphs were only detected among strains of $T$. benhamiae. Trichophyton japonicum and T. concentricum strains exhibited only the MAT1-1-1 idiomorph, while T. europaeum comprised strains characterized by the presence of the MAT1-2-1 idiomorph. Only " $T$. europaeum" strain IHEM 25139 showed MAT1-1-1 idiomorph.

The $\boldsymbol{T}$. erinacei clade comprises three species: T. erinacei, an agent of mycoses in hedgehogs (genera Erinaceus, Aterelix); T. verrucosum, an agent of cattle ringworm; and T. eriotrephon, with poorly known ecological characteristics (Figure 2). All analyzed isolates of $T$. erinacei and $T$. verrucosum presented the MAT1-2-1 idiomorph, while T. eriotrephon exhibited only the MAT1-1-1 idiomorph.

The T. bullosum clade contains three human isolates of the African race of Arthroderma benhamiae, and T. bullosum is a causal agent of dermatomycoses in horses and donkeys. Isolates of the African race apparently represent an independent taxonomic entity, and we propose the name $T$. 
africanum for this species (Figure 2). Both MAT gene idiomorphs were detected in T. africanum, while T. bullosum isolates exhibited only the MAT1-1-1 idiomorph.

\section{Analysis of the T. benhamiae clade with newly designed microsatellite markers}

A total of 160 microsatellite markers with di- or trinucleotide repeats and motifs longer than eleven repetitions were extracted from the available genome of T. europaeum CBS 112371 using WebSat software (Martins et al. 2009). The number of repeats was inferred by subtracting the known length of the flanking sequence from the total amplicon length. Only 24 regions contained the required repeat and showed length polymorphism in the microsatellite region and an absence of polymorphism in the flanking region. A total of ten markers with an even distribution in the genome and different lengths (for the purpose of multiplexing) were selected for the final analysis (Table 2). The Simpson's diversity index calculated for particular loci yielded values ranging from 0.34 (TC20 locus) to 0.59 (TAG16 locus). The whole panel consisting of ten markers yielded a diversity index of 0.77 (Table S4).

This newly developed microsatellite typing scheme was applied to a total number of 318 isolates belonging to the T. benhamiae clade. Forward primers of all loci were marked with fluorescent dye and arranged in a multiplex panel (Table 2). The highest number of alleles was found at the TAG16 locus, followed by the CT21b locus. In contrast, the fewest alleles were found in at the AG18 (n=5) and TC20 $(n=5)$ loci. The remaining loci included 7-9 alleles (Table S4). All loci were successfully amplified in all examined strains (null alleles were not found). The dependence of genotypic diversity on the number of loci showed that a sufficient number of markers was used to resolve the population structure of the $T$. benhamiae clade. It was apparent from the curves (Figure 4) that genetic diversity would not increase significantly with the addition of more markers.

The software STRUCTURE was used to determine how many groups were included in the dataset. The highest $\Delta \mathrm{K}$ value was observed at $\mathrm{K}=6$, and a much lower peak was present at $\mathrm{K}=4$ (Figure 5). The estimated population structure inferred from this analysis is shown in Figure 5. The analysis revealed a total of 41 genotypes among $T$. benhamiae clade isolates clustering into six clusters (C1-C6).

The distribution of the isolates into clusters was correlated with their geographic distribution and main primary hosts (Figure 6). The cluster C1 was found most abundantly in Europe and was associated with guinea pigs. These isolates are responsible for the current outbreak of infections in Central Europe and consists of yellow phenotype strains. We propose the name T. benhamiae var. luteum for this cluster. Clusters C2 and C3 comprised white phenotype strains from North America isolated mostly from dogs and characterized by highly variable microsatellite data (T. benhamiae var. benhamiae). Cluster C4 (T. japonicum) comprised the majority of strains from Japan analysed in this study and some European strains (rabbits, guinea pigs and human infections contracted from them). Cluster C5 (T. europaeum) comprised strains from Europe (infections mostly contracted from guinea pigs). The isolate IHEM 25139 was assigned to $T$. europaeum but its haplotype was intermediate 
between T. europaeum and T. japanicum (alleles CT21 and CT21b were characteristic of T. japonicum, while the remaining 8 alleles were from $T$. europaeum). Cluster C6 was represented by three human isolates of $T$. concentricum from tropical regions.

The clustering based on the microsatellite data was correlated with MAT gene distribution and single-gene DNA data ( $t u b b$ gene was exluded due lack of variability in $T$. benhamiae clade) (Figure 7, Figure S6). It is evident from the visualisation that clustering of isolates according to the single-gene genotype and MAT idiomorphs was in general agreement with microsatellite data and proposed species hypothesis. However, the clusters C1-C3 are not supported by any DNA locus sequences in study and are only distinguishable by microsatellites. Trichophyton benhamiae var. luteum (C1) was characterized by low variability of microsatellite data and exclusively consisted of isolates with MAT11-1 idiomorph. The isolates of $T$. benhamiae var. benhamiae cluster C2 were exclusively of the MAT12-1 idiomorph, while those of cluster C3 were exclusively of the MAT1-1-1 idiomorph. Despite obvious phenotypic and population genetic differences between $T$. benhamiae var. benhamiae and T. benhamiae var. luteum, these two varieties are not distinguishable by any of the DNA sequence markers used in this study. The only detected DNA sequence variant, represented by a single substitution in the tefl- $\alpha$ gene, did not correspond to the two varieties delimited by microsatellite markers. This substitution probably constitutes an incomplete lineage sorting phenomenon (Figure 7).

\section{Genetic diversity and population structure analysis of $T$. benhamiae clade}

Population characteristics were calculated from microsatellite data to test significance of clonal expansion versus recombination, and genetic diversity within clusters. Besides the inability to reproduce sexually due to missing opposite mating type in most of species, the clonality is indicated by the screwed distribution of pairwise differences between individuals (Figure 8). Consequently, all populations are genetically uniform which is evident from low value of Nei's gene diversity (D) (Table S5) that ranged from 0.02 in T. benhamiae var. luteum to 0.145 T. benhamiae var. benhamiae. The low Nei's genotype diversity $(\mathrm{Dg}=0.35)$ of $T$. benhamiae var. luteum compared to other taxa reflects the fact that the population consisted of several abundant clones (Table S5). Asexual reproduction prevails in all populations for long time which is supported by the low effective number of genotype $\left(\mathrm{G}_{\text {eff }}\right)$ values that were significantly lower than observed number of genotypes (Table S5). The exception was $T$. benhamiae var. benhamie cluster C2 (Table S5). However, recombination in cluster C2 was not confirmed by calculation of index of association $\left(\mathrm{I}_{\mathrm{A}}\right)$ (Table S5), possibly due to low number of samples available. The recombination was not rejected only in T. europaeum population according according to $\mathrm{I}_{\mathrm{A}}$ on significance level $\mathrm{p}<0.05\left(\mathrm{I}_{\mathrm{A}}=0.24, \mathrm{p}<0.0042\right)$ (Figure 9, Table S5).

To test cluster-specific differences, AMOVA was performed on the microsatellite data. The diversity between six clusters contributed to a total variability of $68.1 \%$, while the diversity within clusters contributed to only $31.9 \%$ ( $\mathrm{p}<0.0001)$. Thus, there is a low level of genetic information exchange between clusters, reflected in a high number of fixed alleles $\left(\mathrm{F}_{\mathrm{ST}}=0.89, \mathrm{G}_{\mathrm{ST}}=0.75\right.$, 
$\mathrm{p}<0.0001)$. Low gene flow levels between $T$. benhamiae var. luteum, $T$. japonicum and T. europaeum demonstrated by these indices could be explained by reproductive isolation despite overlapping hosts (e.g. guinea pigs) and geographic distributions. This could be caused by pre- or postzygotic reproductive barriers, or absence of terrestrial reservoir for sexual reproduction.

Trichophyton concentricum and T. benhamiae var. benhamiae cluster C3 shared the greatest number of alleles in common $\left(\mathrm{F}_{\mathrm{ST}}=0.451, \mathrm{G}_{\mathrm{ST}}=0.49\right)$. The lowest number of shared alleles was found between T. benhamiae var. luteum and all other clusters $\left(\mathrm{F}_{\mathrm{ST}}=0.90-0.95\right.$; Table $\left.\mathrm{S} 6\right)$. The strongly fixed set of unique alleles in T. benhamiae var. luteum indicates low or no gene flow between this cluster and the remaining clusters. Relatively low DW index value ( $\mathrm{DW}=0.06$; Table S5) indicate recent origin of T. benhamiae var. luteum. On the other hand, high DW values in other taxa indicate long-term isolation due to accumulation of unique alleles (Table S5).

\section{Phenotypic studies}

Initially, the phenotype of all isolates was recorded on malt extract agar (MEA). It was observed that the morphotypes within the $T$. benhamiae clade generally corresponded to the clusters delimited by microsatellite analysis. Notable exceptions were the strains showing signs of degeneration (poorly sporulating, white, cottony colonies usually producing no pigments). Such a phenotype is commonly described in dermatophytes and indicates degeneration, usually caused by long-term strain passaging and preservation. These strains were excluded from further phenotype analyses. At least five strains (if available) from each group were selected, and their phenotypes were analysed on three cultivation media (Figure 10). Growth rates were recorded at three temperatures (Figure 11), and micromorphology was measured on MEA (Figure 12). Cultivation on MEA and potato dextrose agar (PDA) promoted sporulation and pigment production most effectively.

Among the taxa from the T. benhamiae clade, the strains of $T$. concentricum and $T$. benhamiae var. luteum were characterized by the slowest growth on all media and at all tested temperatures (Figure 11). No sporulation was observed in the T. concentricum strains examined in this study. Overall, poor sporulation, the production of intense yellow pigmentation as the colony reverse colour and the absence of macroconidia and spiral hyphae were characteristic of $T$. benhamiae var. luteum (yellow phenotype strains of $T$. benhamiae). All three remaining species from the $T$. benhamiae clade produced both microand macroconidia and whitish colonies, usually with a brownish, red-brown or red colony reverse colour (white phenotype strains of $T$. benhamiae). Trichophyton benhamiae var. benhamiae grew more rapidly at $25^{\circ} \mathrm{C}$ than the other species from this clade (Figure 11) and exhibited larger microconidia on average (Figure 12). The obverse colony colour was whitish or showed a brownish tint, and red-brown pigmentation on the reverse side was commonly arranged into sectors (Figure 10). The growth parameters and micromorphology of T. japonicum and T. europaeum were very similar (Figure 11, Figure 12), and all strains extensively sporulated. 

characterized by relatively long microconidia (comparable to those of $T$. benhamiae var. benhamiae) growing on unbranched or loosely branched conidiophores. Compared to T. africanum, the conidiophores of $T$. benhamiae clade members were either poorly differentiated from vegetative hyphae

\section{5} (conidia sessile on the hyphae) or short with many lateral branches under the top (branched in a pyramidal pattern, grape-like). A more detailed differential diagnosis of particular species with their relatives is included in the Notes in the Taxonomy section.

The ANOVA was performed on microconidia width, length and growth rates (MEA, SAB, PDA at 25,30 and $37^{\circ} \mathrm{C}$ ), followed by a post hoc analysis using Tukey's HSD pairwise comparisons based on the mean values for each strain and a confidence interval of 0.95. All growth rate variables and conidium size variables were strongly correlated. The variables from the two groups can therefore be used interchangeably (Figure 13). The analysis showed that there were statistically significant differences between $T$. benhamiae clade species according to any combination of characteristics, including conidia size and growth rates $(\mathrm{p}<0.001)$. Furthermore, growth rates measured at $25{ }^{\circ} \mathrm{C}$ on MEA or PDA can be used independently to distinguish the majority of species $(\mathrm{p}<0.001)$ (Figure 13, Figure S7, Table S7). Variables such as microconidium length (Table S8) and width (Table S9) can also be used independently to distinguish particular species, except for $T$. japonicum and $T$. benhamiae var. luteum, which cannot be differentiated at the specified significance level.

\section{MALDI-TOF mass spectrometry}

Representative isolates of each species from the $T$. benhamiae clade were analysed using MALDI-TOF mass spectrometry; T. africanum isolates were also included for comparison (Figure 14). All samples could be measured very well and delivered high quality (peak rich) MALDI spectra. In the mass range between approximately 5900-6200 m/z (as an representative example), the MALDI-TOF mass spectra were very similar both between and within all groups, and differentiation of the groups was not possible within this range. In contrast to this high similarity, several specific peaks could be found for all analysed taxa the entire mass range of approximately 4000 to 12,000 m/z (Figure 14). Trichophyton africanum significantly differed from all of the samples in many peaks in its spectrum (Figure 14A). Trichophyton benhamiae var. luteum and T. benhamiae var. benhamiae shared peaks at 7150 and 7745 $\mathrm{m} / \mathrm{z}$ in their mass spectra but different peaks at 4112 and $4680 \mathrm{~m} / \mathrm{z}$, which are typical of var. luteum, and 6515 and $6530 \mathrm{~m} / \mathrm{z}$, which are typical of var. benhamiae (Figure 14C-D). Both mentioned species differ from T. europaeum and T. japonicum in the absence of a peak at $7150 \mathrm{~m} / \mathrm{z}$ (data not shown). Trichophyton europaeum differed from T. japonicum in the presence of a peak at $7745 \mathrm{~m} / \mathrm{z}$ and the absence of a peak at $7715 \mathrm{~m} / \mathrm{z}$ (Figure 14B). Trichophyton concetricum differed from both $T$. benhamiae varieties in its peaks at 4770, 6435 and $7145 \mathrm{~m} / \mathrm{z}$ (Figure 14D) and also differed from the rest of the samples in several peaks. To prove the general applicability of the here presented MALDI peaks more 
strains of the mentioned species / varieties should be analyzed in the future and incorporated into the presented MALDI-based differentiation model.

\section{TAXONOMY}

\section{Trichophyton benhamiae clade}

Trichophyton benhamiae (Ajello \& S.L. Cheng) Y. Gräser \& de Hoog [Index Fungorum 356: 2. 2018] var. benhamiae (automatically generated; Art. 26.3 [Turland et al. (2018)]) - MycoBank XXXX; Figure 15

Vegetative hyphae smooth, septate, hyaline, 1.5-4 $\mu \mathrm{m}$ diam (mean $\pm \mathrm{sd} ; 2.5 \pm 0.7$ ). Conidiophores poorly differentiated from vegetative hyphae, mostly unbranched, conidia sessile or born on short lateral branches; pyramidally branches conidiophores less common and with sparse branching. Microconidia abundant, pyriform to clavate, truncate, $2.5-6(3.8 \pm 0.5) \times 1.6-3.5(2.6 \pm 0.4) \mu \mathrm{m}$. Macroconidia sparse to abundant, cylindrical or elongated fusiform, with pointed or rounded ends, easily disintegrate into fragments with truncate ends, developing intercalary or terminally on vegetative hyphae, frequently released with short to long mycelial fragments at one or both ends, predominantly 3-10-septate (median 8), 23-82 $(59.2 \pm 15.5) \times 4.5-7.5(6.1 \pm 0.8) \mu \mathrm{m}$. Chlamydospores present. Spiral hyphae absent or rare. Heterothallic. Sexual state fide Ajello \& Cheng (1967) and Čmoková (2015): Cleistothecia white to yellowish-white, covered with dichotomously branched peridial hyphae and spiral appendages. Peridial hyphae composed of asymmetrical peridial cells, dumb-bell shaped, echinulate, 8.5-10.5 (9.1 $\pm 1.8) \mu \mathrm{m}$ in length, $2.5-4.5(2.8 \pm 0.7) \mu \mathrm{m}$ in width at enlarged ends, internode width $2-4 \mu \mathrm{m}(2.4 \pm$ 1.2); intercalary conidia sparse, cylindrical or barrel-shaped. Asci globose, eight-spored, ascospores ovate, hyaline to pale yellow, longer dimension up to $3 \mu \mathrm{m}$, shorter dimension up to $2 \mu \mathrm{m}$.

Culture characteristics - (Colonies in $7 \mathrm{~d}$ at $\left.25^{\circ} \mathrm{C}\right)$ Colonies on SAB $28-34 \mathrm{~mm}$ diam $(\varnothing=32 \mathrm{~mm})$, White (\#F2F3F4), velvety to powdery, centrally raised, radially furrowed in some strains, edge diffuse, reverse Pale Orange Yellow (\#FAD6A5) to Light Orange Yellow (\#FBC97F) in the marginal part, Vivid Orange (\#F38400) to Deep Brown (\#593319) in the center. Colonies on MEA 30-35 mm diam ( $\varnothing=34 \mathrm{~mm}$ ), velvety to granular, Pale Yellow-gray (\#C7ADA3) to Light Yellow (\#FAD6A5), umbonate, edge diffuse, reverse Pale Orange Yellow (\#FAD6A5) to Brilliant Orange Yellow (\#FFC14F), red pigment produced in sectors by some strains - Deep Reddish Orange (\#AA381E). Colonies on PDA 27-32 mm diam ( $\varnothing=30 \mathrm{~mm}$ ), White (\#F2F3F4) to Light Yellow (\#FAD6A5), velvety to granular, centrally raised, occasionally with filamentous sectors, reverse Pale Orange Yellow (\#FAD6A5) to Brilliant Orange Yellow (\#FFC14F), red pigment produced in sectors by some strains Deep Reddish Orange pigment (\#AA381E). Colonies in $7 \mathrm{~d}$ at $30{ }^{\circ} \mathrm{C}$ grow faster than at $25^{\circ} \mathrm{C}$ : SAB $37-45 \mathrm{~mm}$ diam $(\varnothing=39 \mathrm{~mm})$; PDA 35-43 $\mathrm{mm}$ diam $(\varnothing=37 \mathrm{~mm})$; MEA $8-43 \mathrm{~mm}$ diam $(\varnothing=40 \mathrm{~mm})$. 
1 Colonies at $37^{\circ} \mathrm{C}$ in $7 \mathrm{~d}$ : SAB $27-39 \mathrm{~mm}$ diam $(\varnothing=30 \mathrm{~mm})$; PDA $30-35 \mathrm{~mm}$ diam $(\varnothing=34 \mathrm{~mm})$; MEA

$2 \quad 30-35 \mathrm{~mm}$ diam $(\varnothing=33 \mathrm{~mm})$.

3

Specimens examined. USA, Missouri, human, Ajello [epitype designated here MBTXXXX, PRM 944659, a dried culture derived from strain IHEM 4710, culture ex-type IHEM 4710 (= CBS $623.66=$ ATCC $16781=$ CABIM $124768=$ CDC X-797 = CECT $2892=$ IMI $124768=$ IP $1064.74=$ NCPF $0410=$ RV 23303 = UAMH 2822]. USA, Urbana, dog, 2009 (USA 3208). USA, Urbana, dog, 2006 (USA 3209); ibid., USA 3216. USA, Urbana, cat, 2006 (USA 3220). USA, Urbana, dog, 2007 (USA 3329). USA, Urbana, dog, 2010 (USA 3350); ibid., USA 3355; ibid., USA 3356. USA, Urbana, chinchilla, 2011 (USA 3360). USA, Urbana, dog, 2011 (USA 3361). USA, Urbana, unknown source, 1991 (USA 3368). USA, Urbana, unknown source, 1989 (USA 3369). USA, Urbana, unknown source, 1997 (USA 3370). USA, Urbana, unknown source, 2001 (USA 3371). USA, Urbana, unknown source, 1996 (USA 3376). USA, Urbana, unknown source, 1995 (USA 3378). IN-VITRO, monoascospore isolate, 1970, M. Takashio [IHEM 3287 = RV 26678; isolate from cross between IHEM 24908 (ex dog, USA) $\times$ IHEM 4710 (ex human, USA)]. IN-VITRO, monoascospore isolate, 1970, M. Takashio [IHEM $3288=$ BER $1464=$ DSM 6916 = JS 83-006 = RV $26680=$ SM $0104=$ VUT $77012=$ CCRC $31780=$ IAM $12705=$ JCM 1886; isolate from cross between IHEM 24908 (ex dog, USA) $\times$ IHEM 4710 (ex human, USA)].

Typification - Ajello \& Cheng (1967) designated the specimen NCDC B765d as a holotype of $T$. benhamiae, and a dried culture with ascomata was generated by crossing the isolates TM-20 (= ATCC $16781=$ IHEM $4710=$ CBS $623.66=$ CABIM $124768=$ CDC X-797 = CECT $2892=$ IMI $124768=$ IP 1064.74 = NCPF 0410 = RV 23303 = UAMH 2822; ex human; MAT1-2-1) $\times$ TM-17 (= ATCC $16782=$ CBS $624.66=$ IHEM $24908=$ RV $23302=$ CDC X-798 = CECT $2893=$ IMI $124769=$ NCPF 411 = UAMH 2823; ex dog; MAT1-1-1). Although this specimen exhibits both sexual and asexual morphs in its life cycle, it is not suitable for the purposes of the recent taxonomy for several reasons. First, it is not clear which of the two cultures contained within the type should be considered the exholotype culture. Additionally, interspecific hybrids can be induced by crossing opposite mating type strains of unrelated species in vitro as shown in previous studies on dermatophytes (Kawasaki et al. 2009, Anzawa et al. 2010, Kawasaki et al. 2010, Kawasaki et al. 2011), and the deposition of a resultant 'hybrid' type could lead to ambiguities. Because it is not possible to recognize which portion of the holotype belongs to a particular isolate, we designated an epitype PRM 944659 (dried culture) derived from the IHEM $4710(=$ CBS $623.66=$ ATCC $16781=$ CABIM $124768=$ CDC X-797 = CECT 2892 $=$ IMI $124768=$ IP $1064.74=$ NCPF $0410=$ RV $23303=$ UAMH 2822).

Distribution and ecology - Trichophyton benhamiae var. benhamiae is a zoophilic dermatophyte, and isolates examined in this study originated from $\operatorname{dogs}(n=8)$, cats (isolate USA 3220), chinchillas (isolate USA 3360) and unknown hosts $(n=6)$. Previously reported cases of human infections were probably 
transmitted from animals (Ajello and Cheng 1967). Another important host of this pathogen is probably the North American porcupine (Erethizon dorsatum) (Takahashi et al. 2008, Needle et al. 2019), a close relative of the guinea pig (Cavia porcellus). Isolates from the North American porcupine exhibited ITS rDNA identical to that of $T$. benhamiae, and their morphology showed characteristics typical of $T$. benhamiae var. benhamiae (Takahashi et al. 2008, Needle et al. 2019). All strains examined here were collected in North America (the in vitro-derived isolates were also based on strains of American origin). A recently reported a Chinese case of tinea faciei, likely contracted from fox, that was probably also caused by T. benhamiae var. benhamiae based on the ITS sequence and morphology of the isolate (Tan et al. 2020).

Notes - The macromorphology of T. benhamiae var. benhamiae most closely resembles those of $T$. europaeum, T. japonicum and T. mentagrophytes in the production of a red-brown pigment on reverse side of colonies and abundant microconidia. It differs from T. europaeum and T. japonicum in its host spectrum and higher growth rates, especially on MEA and PDA at $25^{\circ} \mathrm{C}$ (Figure 11). Macroconidia of T. benhamiae var. benhamiae are usually more abundantly produced compared to T. europaeum and T. japonicum, and they are most frequently cylindrical or elongated fusiform with terminal fragments of vegetative hyphae. Closely related $T$. concentricum differs significantly in its ecology. It is an anthropophilic species occurring in tropical regions, grows very slowly, produces cerebriform colonies without red-brown pigment on the colony reverse and usually does not sporulate. Trichophyton behamiae var. luteum is also strikingly different in its host spectrum (mostly guinea pigs), distribution (mainly Europe) and morphology (slow growth, yellow pigmentation, relatively poor sporulation, absence of macroconidia). Trichophyton benhamiae var. benhamiae does not produce intense yellow pigment on SAB supplemented with chloramphenicol and cycloheximide and MEA, in contrast to $T$. benhamiae var. luteum. The ratio of MAT1-1-1 and MAT1-2-1 strains was 14:5.

Trichophyton benhamiae (Ajello \& S.L. Cheng) Y. Gräser \& de Hoog [Index Fungorum 356: 2. 2018] var. luteum Cmokova \& Hubka, var. nov. - MycoBank XXXX; Figure 16

Etymology. Refers to the bright yellow colonies produced especially on SAB with chloramphenicol and cycloheximide and MEA.

Vegetative hyphae smooth, septate, hyaline, 1-3.5 $\mu \mathrm{m}$ diam (mean $\pm \mathrm{sd}$ : $1.9 \pm 0.5$ ). Conidiophores branched in a pyramidal (grape-like) pattern, sometimes poorly differentiated from vegetative hyphae, unbranched or poorly branched, conidia sessile or born on short lateral branches. Microconidia sparse to abundant, pyriform, less commonly clavate, $2.5-4.9(3.2 \pm 0.4) \times 1.5-3.4(2.1 \pm 0.3) \mu \mathrm{m}$. Macroconidia not observed in any of the isolates examined. Chlamydospores were not observed. Spiral hyphae not observed. Sexual morph unknown. 
2 Culture characteristics - (Colonies in $7 \mathrm{~d}$ at $\left.25^{\circ} \mathrm{C}\right)$ colonies on SAB $10-20 \mathrm{~mm} \operatorname{diam}(\varnothing=13 \mathrm{~mm})$,

3 White (\#F2F3F4) to Yellowish White (\#F0EAD6), velvety, flat with radially furrowed center, edge

4 filliform, reverse Vivid Yellow (\#F3C300). Colonies on MEA 6-17 mm diam ( $\varnothing=12 \mathrm{~mm})$, Pale Yellow

5 (\#F3E5AB), filamentous, flat, edge filliform, reverse Light Yellow (\#F8DE7E) to Vivid Yellow

6 (\#F3C300). Colonies on PDA 9-17 mm diam $(\varnothing=13 \mathrm{~mm}$ ), Light Yellow (\#F8DE7E) to Pale Yellow

7 (\#F3E5AB), velvety, flat, radially furrowed, edge filliform, reverse Brilliant Orange Yellow (\#FFC14F)

8 to Vivid Yellow (\#F3C300). Colonies at $30^{\circ} \mathrm{C}$ in $7 \mathrm{~d}$ : SAB $15-26 \mathrm{~mm}$ diam $(\varnothing=21 \mathrm{~mm})$; PDA $18-22$

$9 \mathrm{~mm} \operatorname{diam}(\varnothing=21 \mathrm{~mm})$; MEA $21-22 \mathrm{~mm}$ diam $(\varnothing=22 \mathrm{~mm})$. Colonies at $37{ }^{\circ} \mathrm{C}$ in $7 \mathrm{~d}: \mathrm{SAB} 15-20 \mathrm{~mm}$ $\operatorname{diam}(\varnothing=18 \mathrm{~mm}) ; \operatorname{PDA} 10-17 \mathrm{~mm} \operatorname{diam}(\varnothing=12 \mathrm{~mm}) ; \operatorname{MEA} 11-13 \mathrm{~mm} \operatorname{diam}(\varnothing=11 \mathrm{~mm})$.

Specimens examined. SWITZERLAND, Lausanne, University Hospital Vaudois, dermatophytosis in human, arm skin (tinea corporis), 2009, M. Monod (PRM 944414, holotype, dried culture; PRM 944415, isotype; culture ex-type IHEM 25068). JAPAN, common degu, 2012 (NUBS 13001). SwITZERLAND, Lausanne, University Hospital Vaudois, human skin, 2009, M. Monod (IHEM 25066). CZECHIA, Prague, guinea pigs (Cavia porcellus), 2014, J. Koubová (KOUB 23); ibid., KOUB 51; ibid., KOUB 77. GERMANY, Berlin, dermatophytosis in human, 2010 (BER 24); ibid., BER 211; ibid., BER 212; ibid., BER 213. CZECHIA, České Budějovice, dermatophytosis in human, 2012 (D126); ibid., D295; ibid., D375; ibid., D417; ibid., D521. GERMANY, Mölbis, dermatophytosis in human, 2015 (DE 200156); ibid., DE 200351; ibid., DE 200465. BELGIUM, Brussels, dermatophytosis in human, 2012 (IHEM 25744); ibid., IHEM 25743; ibid., IHEM 25742; ibid., IHEM 25466; ibid., IHEM 25745. CZECHIA, Prague, dermatophytosis in human, 2012 (CCF 4849); ibid., CCF 4850; ibid., CCF 4851; ibid., CCF 4852. All 236 strains examined in this study are listed in Table S1.

Distribution and ecology — Trichophyton benhamiae var. luteum is a zoophilic species with the guinea pig as the main host (Hubka et al. 2018c). It is widely distributed in Europe, but it has also been detected in common degu (Octodon degus) in Japan (Hiruma et al. 2015) and was recently isolated from human dermatophytosis in Brazil (de Freitas et al. 2019, Grisólia 2019).

Notes - The macromorphology of T. benhamiae var. luteum resembles that of Microsporum canis in the production of intense yellow pigmentation as the colony reverse colour. However, $M$. canis usually produces abundant spindle-shaped macroconidia, which are absent in T. benhamiae var. luteum. The differentiation of sterile $M$. canis isolates may be more difficult but is possible according to its higher growth parameter values. In addition, these species differ in their main hosts, which are cats and dogs in M. canis and guinea pigs in T. benhamiae var. luteum. The closely related anthropophilic species $T$. concetricum differs in its ecology, colony characteristics (no yellow pigment produced) and 
microscopic characteristics (usually no sporulation). Other taxa from the T. benhamiae clade differ in showing higher growth rates (Figure 11), the production of red/brown pigments and the production of macroconidia, which are absent in $T$. benhamiae var. luteum. In addition to these differences, $T$. benhamiae var. luteum can be clearly distinguished from $T$. benhamiae var. benhamiae and other species in the T. benhamiae clade by microsatellite data (Figures 5-6) and MALDI-TOF MS spectra (Figure 14).

The European strains of $T$. benhamiae var. luteum $(\mathrm{n}=236)$ examined here were predominantly obtained from humans ( $72 \%$ from females and $\sim 28 \%$ from males, median age 12 years) who mostly reported contact with guinea pigs; the remaining strains were recovered from animals (guinea pigs and common degu) (Table S1). The human infections mostly manifested as highly inflammatory tinea corporis, tinea faciei and tinea capitis (Fig. 17). By contrast, infected animals were mostly symptomless. Symptomatic guinea pigs usually showed localized lesions with scaling and crusting or alopecia located predominantly on the head, less frequently on the other body parts (Fig. 17). Green fluorescence of infected tissues may be observed under Wood's light in some strains, similar to M. canis (Skořepová et al. 2014). Only the MAT1-1-1 idiomorph was detected in the T. benhamiae var. luteum isolates examined here.

Trichophyton concentricum R. Blanch., Traité de Pathologie Générale 2: 916. 1896 — Figure 18

Vegetative hyphae smooth, septate, frequently inflated, occasionally with knob-like terminations, often proliferating in a zigzag pattern, hyaline, $1.5-4 \mu \mathrm{m}$ diam (mean $\pm \mathrm{sd} ; 2.7 \pm 0.7$ ). Chlamydospores common, usually globose or ovate, intercalar, terminal or in short chains. Conidiophores, conidia, pectinate hyphae and favic chandeliers were not observed among the examined strains. Sexual morph unknown.

Culture characteristics - (Colonies in $7 \mathrm{~d}$ at $\left.25{ }^{\circ} \mathrm{C}\right)$ Colonies on SAB $6-16 \mathrm{~mm}$ diam $(\varnothing=11 \mathrm{~mm})$, Pale Orange Yellow (\#FAD6A5) to Pale Yellowish Pink (\#ECD5C5), membranous to slightly velvety, raised, umbonate or cerebriform, deeply furrowed, edge filiform or lobate, reverse Light Orange Yellow (\#FBC97F). Colonies on MEA 9-16 mm diam ( $\varnothing=15 \mathrm{~mm}$ ), Pale Orange Yellow (\#FAD6A5) to Pale Yellowish Pink (\#ECD5C5), membranous to slightly velvety, umbonate, edge filiform, reverse Light Orange Yellow (\#FBC97F) to Brilliant Orange Yellow (\#FFC14F), Vivid Yellow (\#F3C300) in narrow centre. Colonies on PDA 5-12 mm diam $(\varnothing=11 \mathrm{~mm}$ ), Pale Orange Yellow (\#FAD6A5) to Pale Yellowish Pink (\#ECD5C5), membranous, raised, deeply furrowed to cerebriform, edge irregular to lobate, reverse Light Orange Yellow (\#FBC97F) to Brilliant Orange Yellow (\#FFC14F), Vivid Yellow (\#F3C300) in narrow centre. Colonies at $30^{\circ} \mathrm{C}$ in $7 \mathrm{~d}$ : SAB $8-20 \mathrm{~mm}$ diam $(\varnothing=16 \mathrm{~mm})$; MEA 8-15 $\mathrm{mm}$ diam $(\varnothing=13 \mathrm{~mm})$; PDA $10-14 \mathrm{~mm} \operatorname{diam}(\varnothing=11 \mathrm{~mm})$. Colonies at $37{ }^{\circ} \mathrm{C}$ in $7 \mathrm{~d}: \mathrm{SAB} 5-14 \mathrm{~mm}$ $\operatorname{diam}(\varnothing=10 \mathrm{~mm})$; MEA 5-13 mm diam $(\varnothing=10 \mathrm{~mm})$; PDA 5-13 $\mathrm{mm}$ diam $(\varnothing=9 \mathrm{~mm})$. 
2 Specimens examined. POLYNESIA, human, 1926, A. Castellani (ex-neotype strain CBS 196.26 = IFO 3 5972). FIJI, human skin, 1963 (CCF 5303 = IHEM 13435 = RV 30442). INDONESIA, Manado, human, arm and trunk skin, 1990, W. Warow (CCF $5302=$ IHEM 5470)

6 Distribution and ecology - Trichophyton concentricum is an anthropophilic species distributed in 7 Oceania, Southeast Asia, and Central and South America. It is a cause of tinea imbricata (tokelau) usually affecting rural indigenous populations. The clinical manifestation is very characteristic and gives human skin ornate appearance due to the presence of concentric squamous plaques (Bonifaz et al. 2004, Pihet et al. 2008, Bonifaz and Vazquez-Gonzalez 2011).

Notes - The morphology of T. concetricum resembles those of the slow-growing species $T$. verrucosum, $T$. bullosum (for differentiation see T. bullosum description) and T. schoenleinii. Closely related species from the $T$. benhamiae clade are easily distinguished from $T$. concetricum by higher growth rates (Figure 11) and relatively abundant sporulation. Differentiation from these species is usually not problematic in practice due to the different host spectra and geographic distributions of these species. Only the MAT1-1-1 idiomorph was detected in the T. concentricum isolates examined here; in contrast, isolates of $T$. verrucosum and T. schoenleinii exclusively show the MAT1-2-1 idiomorph (Kano et al. 2014, Kosanke et al. 2018).

Trichophyton concetricum usually grows as a sterile mycelium in culture; however, the production of clavate microconidia and smooth-walled macoconidia has been observed by some authors (Rippon 1988, Pihet et al. 2008), while favic chandeliers and pectinate hyphae ("antler" tips) are more frequently reported (Dvořák and Otčenášek 1969, Bonifaz et al. 2004). We did not observe these structures in any of the isolates examined.

Trichophyton europaeum Cmokova \& Hubka, sp. nov. — MycoBank XXXX; Figure 19

Etymology. Refers to the origin of the examined strains.

Vegetative hyphae smooth, septate, hyaline, 1-3 $\mu \mathrm{m}$ diam (mean \pm sd: $1.9 \pm 0.3$ ). Conidiophores branched in a pyramidal (grape-like) pattern or poorly differentiated from the hyphae and represented by conidiogenous hyphae with sparse to numerous short lateral branches. Microconidia abundant, sessile on lateral or terminal branches, pyriform to clavate, $2.5-3.9(3 \pm 0.3) \times 1.5-2.8(2.1 \pm 0.2) \mu \mathrm{m}$. Macroconidia rare to sparse, born terminally on hyphae, rare to sparse, usually consisting of 2-7 cells $($ median $=4)$ with an unequal diameter, 45-76 $(51.2 \pm 7.3) \times 3-10.5(5 \pm 1.3) \mu \mathrm{m}$, elongated, clavate, less frequently fusiform, with a tapering rounded apex and truncate end, cylindrical fragments of macroconidia common, macroconidia consisting of irregular and bloated cells common. 
Chlamydospores present. Spiral hyphae absent to rare in 14-d-old cultures, usually consisting of one to

several coils. Sexual morph unknown, pseudo-ascomata are formed by some isolates after prolonged incubation.

Culture characteristics - (Colonies in $7 \mathrm{~d}$ at $\left.25^{\circ} \mathrm{C}\right)$ Colonies on SAB $24-29 \mathrm{~mm}$ diam $(\varnothing=25 \mathrm{~mm})$, White (\#F2F3F4), velvety to floccose, flat, in some strains with radially wrinkled or elevated center, edge filiform, difuse or entire, reverse Brilliant Yellow (\#FADA5E), to Deep Orange Yellow (\#C98500). Colonies on MEA 20-30 mm diam ( $\varnothing=26 \mathrm{~mm}$ ), White (\#F2F3F4) to Light Yellow (\#F8DE7E), velvety, floccose to coarsely granular, flat with an umbonate center, edge entire to difuse, reverse in shades of brown [Strong Orange Yellow (EAA221) to Deep Orange (\#BE6516)] or red [Vivid Reddish Orange (\#E25822) to Vivid Red (\#BE0032)]. Colonies on PDA 19-23 mm diam ( $\varnothing=21 \mathrm{~mm}$ ), White (\#F2F3F4) to Light Yellow (\#F8DE7E), velvety, floccose to coarsely granular, flat with an umbonate center, edge irregular, lobate dendritic, reverse yellow (\#F3C300) in the marginal part, Strong Orange Yellow (\#EAA221) to Deep Orange (\#F38400) in the center. Colonies at $30{ }^{\circ} \mathrm{C}$ in $7 \mathrm{~d}$ : SAB 32-37 mm diam ( $\varnothing=35 \mathrm{~mm}$ ); PDA 29-31 mm diam ( $\varnothing=30 \mathrm{~mm}$ ); MEA 32-39 $\mathrm{mm}$ diam $(\varnothing=36 \mathrm{~mm})$. Colonies at $37^{\circ} \mathrm{C}$ in $7 \mathrm{~d}$ : SAB $23-31 \mathrm{~mm} \operatorname{diam}(\varnothing=28 \mathrm{~mm})$; PDA $24-31 \mathrm{~mm}$ diam $(\varnothing=29 \mathrm{~mm})$; MEA $20-30 \mathrm{~mm} \operatorname{diam}(\varnothing=27 \mathrm{~mm})$.

Specimens examined. SWITZERLAND, Lausanne, guinea pig (Cavia porcellus), 2008, M. Monod (PRM 944419, holotype, dried culture; ex-holotype culture IHEM 22725). FRANCE, Lyon, guinea pig, 1963 (IHEM 25139 = CBS $806.72=$ RV 14387). SWITZERLAND, Lausanne, human dermatophytosis (contact with guinea pig), 2002, M. Monod (IHEM 20159 = CBS 112370); ibid., IHEM 25062. SwITZERLAND, Lausanne, human dermatophytosis (contact with guinea pig), 2007, M. Monod (IHEM 25064). SWITZERLAND, Lausanne, tinea corporis (contact with guinea pig), 2010, M. Monod (IHEM 25075). SWITZERLAND, Lausanne, tinea faciei, 2011, Monod (HEM 25076). SwITZERLAND, Lausanne, guinea pig, 2002, M. Monod (IHEM 22723). Czechia, Ostrava, 5-years girl, 2012, S. Dobiášová (CCF 4917). Czechia, Prague, human dermatophytosis (tinea faciei), 2012, M. Skořepová (CCF 4848). CzECHIA, Bylany, dermatophytosis in human (tinea corporis), M. Skořepová (CCF 4853). All 40 strains of $T$. europaeum examined in this study are listed in Table S1.

Distribution and ecology - Trichophyton europaeum is a zoophilic species that is widely distributed in guinea pigs in Europe (Fumeaux et al. 2004, Fréalle et al. 2007, Symoens et al. 2013) but is currently less prevalent than T. benhamiae var. luteum. The species has also been reported from guinea pigs in Japan (Takeda et al. 2012) and human dermatophytosis in Iran (Rezaei-Matehkolaei et al. 2016). Dermatophytosis in horses reported in Egypt is an unusual finding (Tartor et al. 2016). 
The European strains of $T$. europaeum $(\mathrm{n}=41)$ examined here were predominantly obtained

7 from humans ( $\sim 80 \%$ from females and $\sim 20 \%$ from males, median age 12 years) who mostly reported contact with guinea pigs (66\%), and the remaining strains were recovered from animals (guinea pigs, $24 \%$ ) or dogs (Table S1). The infections mostly manifested as tinea corporis (79\%) and tinea faciei (21\%). Only the MAT1-2-1 idiomorph was detected in the T. europaeum isolates examined here, with the exception of the IHEM 25139 strain.

Notes - The morphology of T. europaeum most closely resembles those of $T$. benhamiae var. benhamiae, T. japonicum and T. mentagrophytes. Trichophyton europaeum shares many morphological characteristics with $T$. japonicum, including the red/brown pigmentation of the colony reverse colour on MEA in some strains, the production of conidiophores branched in a pyramidal pattern and abundant sporulation. The ratio of MAT1-1-1 and MAT1-2-1 strains in the T. europaeum strains examined here was 1:39; by contrast, all T. japonicum strains exhibited only the MAT1-1-1 idiomorph (Figures 3, 7). These two species can be reliably differentiated only by means of molecular methods (ITS and gapdh gene sequences, microsatellite markers, MALDI-TOF MS). T. benhamiae var. benhamiae differs from T. europaeum and T. japonicum in its host spectrum, higher growth rates, especially on MEA and PDA at $25^{\circ} \mathrm{C}$ (Figure 11) and macroconidia characteristics. The differentiation of $T$. mentagrophytes from T. europaeum and T. japonicum is sometimes difficult by morphological methods. In general, the obverse of T. mentagrophytes colonies is more intensively coloured in shades of yellow-brown to brown, and the colony reverse colour is usually dark brown. T. mentagrophytes isolates usually produce abundant spiral hyphae, which are rather rare in T. europaeum and T. japonicum after 2 weeks. To differentiate T. europaeum from other species, see the descriptions of T. benhamiae var. benhamiae and T. benhamiae var. luteum.

Trichophyton japonicum Cmokova \& Hubka, sp. nov. — MycoBank XXXX; Figure 20

Etymology. Refers to the origin of the majority of the examined strains.

Vegetative hyphae smooth, septate, hyaline, $1.5-4 \mu \mathrm{m}$ diam (mean \pm sd: $2.5 \pm 0.6$ ). Conidiophores usually poorly differentiated from hyphae and represented by conidiogenous hyphae with sparse to numerous short lateral branches; conidiophores branched in a pyramidal (grape-like) pattern relatively rare. Microconidia abundant, born terminally on hyphae, pyriform to clavate, $2.5-5(3.2 \pm 0.4) \times 1.5-$ $3.6(2.3 \pm 0.3) \mu \mathrm{m}$. Macroconidia rare to abundant, born terminally on hyphae, sparse to abundant depending on the isolate, consisting of 3-8(-12) cells (median $=5), 11-79(55.2 \pm 12.4) \times 5-11(6.8 \pm$ 1.5) $\mu \mathrm{m}$, elongated, cigar-shaped, clavate, with a tapering rounded apex and truncate end, macroconidia consisting of irregular and bloated cells common, long macroconidia easily disintegrate into cylindrical 
fragments. Chlamydospores present. Spiral hyphae absent to sparse in 14-d-old colonies. Sexual morph unknown.

Culture characteristics - (Colonies in $7 \mathrm{~d}$ at $\left.25^{\circ} \mathrm{C}\right)$ Colonies on SAB $16-36 \mathrm{~mm}$ diam $(\varnothing=23 \mathrm{~mm})$, White (\#F2F3F4) to Pale Yellowish Pink (\#ECD5C5), velvety to floccose, flat with sligtly elevated and furrowed center, edge entire to diffuse, reverse Light Orange (\#FAB57F) to Vivid Orange Yellow (\#F6A600) in the marginal part, in some strains Deep Orange Yellow (\#C98500) center. Colonies on MEA 18-30 mm diam ( $\varnothing=26 \mathrm{~mm}$ ), White (\#F2F3F4), Light Yellow (\#F8DE7E) to Pale Yellowish Pink (\#ECD5C5), floccose to granular, flat, sometimes with an umbonate center, frequently with concentric ring pattern, margin entire to diffuse, reverse Deep Orange (\#BE6516), Strong Reddish Brown (\#882D17) to Vivid Red (\#BE0032). Colonies on PDA 16-27 mm diam ( $\varnothing=23 \mathrm{~mm}$ ), White (\#F2F3F4) to Pale Yellowish Pink (\#ECD5C5), floccose to granular, occasionally with cottony sectors, flat or umbonate, margin entire, reverse Deep Orange (\#BE6516), Strong Reddish Brown (\#882D17) to Vivid Red (\#BE0032). Colonies at $30{ }^{\circ} \mathrm{C}$ in $7 \mathrm{~d}$ : SAB 32-45 mm diam $(\varnothing=38 \mathrm{~mm}$ ); MEA 28-37 $\mathrm{mm} \operatorname{diam}(\varnothing=33 \mathrm{~mm})$; PDA $26-35 \mathrm{~mm}$ diam $(\varnothing=30 \mathrm{~mm})$. Colonies at $37^{\circ} \mathrm{C}$ in $7 \mathrm{~d}$ : SAB $21-38 \mathrm{~mm}$ $\operatorname{diam}(\varnothing=26 \mathrm{~mm})$; MEA 32-37 mm diam $(\varnothing=35 \mathrm{~mm})$; PDA 30-35 mm diam $(\varnothing=33 \mathrm{~mm})$.

Specimens examined. SPAIN, human, 1963, P. Miguens (PRM 944416, holotype, dried culture; PRM 944417, isotype; culture ex-type IHEM $17701=$ ATCC $28063=$ CBS $807.72=$ CECT $2894=$ RV 14988). BelgiUM, dog, 1971, De Vroey (IHEM $4030=$ ATCC $28067=$ CBS $809.72=$ RV 28105). JAPAN, rabbit, 2009 (NUBS 09011). JAPAN, Saitama, human, 2000 (VUT 00003-2). JAPAN, Saitama, rabbit, 1999 (VUT 00002). JAPAN, Saitama, rabbit, 2000 (VUT 00003). JAPAN, human, 2013 (NUBS12001). JAPAN, Hyogo, rabbit, 1997 (VUT 97010). JAPAN, unknown source (JPN3). JAPAN, unknown source, unknown (JPN6). JAPAN, human, unknown (NUBS13002). CZECHIA, human, tinea corporis, 2013, N. Mallátová (D 35). CZECHIA, human, tinea corporis, 2011, S. Dobiášová (DMF 3061). CZECHIA, human, tinea corporis, 2012, S. Dobiášová (DMF 2446); ibid., DMF 3031. CZECHIA, human, tinea corporis, 2013, S. Dobiášová (DMF 1658). CZECHIA, guinea pig (Cavia porcellus), 2014, J. Koubková (KOUB 63). CZECHIA, Pardubice, human, tinea corporis, 2011, K. Mencl (ME 961). CZECHIA, Prague, human, tinea corporis, 2012, P. Lysková (PL 1773).

Distribution and ecology — Trichophyton japonicum is a zoophilic species occurring mostly in rabbits and guinea pigs. The species is widely distributed in Japan (mostly in rabbits) (Takeda et al. 2012, Kimura et al. 2015). In Europe it occurs mostly in guinea pigs and less frequently in rabbits and other hosts. In guinea pigs it is less common than T. benhamiae var. luteum and T. europaeum (see discussion). It has also been detected in Thailand (Vu et al. 2019), South Korea (P.-L. Sun, pers. comm.) and Iran (GenBank JX413540; unpublished record). 
The European and Japan strains of T. japonicum $(\mathrm{n}=19)$ examined here were predominantly obtained from humans ( $63 \%$ from females and $\sim 37 \%$ from males, median age 15 years) who mostly reported contact with rabbits, guinea pigs and dogs. The remaining strains were recovered from the mentioned animals (Table S1). The infections mostly manifested as tinea corporis (trunk skin $38 \%$, extremities $63 \%$ ). Only the MAT1-1-1 idiomorph was detected in the T. japonicum isolates examined here.

Notes - For the differentiation of $T$. japonicum from similar species, see the description of $T$. europaeum. Only the MAT1-1-1 idiomorph was detected in all examined strains, by contrast all $T$. europaeum strains exhibited the MAT1-2-1 idiomorph except for strain IHEM 25139.

\section{Trichophyton erinacei clade}

Trichophyton erinacei (J.M.B. Sm. \& Marples) Quaife, J. Clin. Pathol. 19: 178. 1966 — Figure 21

Vegetative hyphae smooth, septate, hyaline, $1-3 \mu \mathrm{m}$ diam (mean $\pm \mathrm{sd}: 1.9 \pm 0.8$ ). Conidiophores usually poorly differentiated from vegetative hyphae, conidiophores branched in a pyramidal (grapelike) pattern present only in some strains, conidia sessile on hyphae or short lateral and terminal branches. Microconidia abundant, mostly clavate or pyriform, 2.9-6.5 (4.3 \pm 2.79$) \times 1.5-3.5(2.7 \pm$ 0.28) $\mu \mathrm{m}$ diam. Macroconidia rare to abunant, predominantly consisting of only two or few cells (intermediate forms between micro- and macroconidia), max. 5-celled (median $=2$ ), clavate, cigarshaped, 6-35 $(11 \pm 4.52) \times 2.5-4.5(3.4 \pm 0.17) \mu \mathrm{m}$; intercalary conidia sparse to abundant, cylindrical, barrel-shaped or irregular. Chlamydospores present. Spiral hyphae not observed. Sexual morph unknown.

Culture characteristics - (Colonies in $7 \mathrm{~d}$ at $\left.25^{\circ} \mathrm{C}\right)$ Colonies on SAB $19-32 \mathrm{~mm}$ diam $(\varnothing=29 \mathrm{~mm})$, White (\#F2F3F4) to Light Orange Yellow (\#F3E5AB) in the centre, flat, finely to coarsely granular, edge difuse, reverse Light Orange Yellow (\#F3E5AB) to Vivid Yellow (\#FADA5E), Deep Reddish Brown (\#882D17) in the centre. Colonies on MEA 19-30 mm diam ( $\varnothing=25 \mathrm{~mm})$, White (\#F2F3F4), flat, finely to coarsely granular, edge difuse, reverse Light Orange Yellow (\#F3E5AB) to Vivid Reddish Orange (\#F38400). Colonies on PDA 11-32 mm diam $(\varnothing=24 \mathrm{~mm})$ White (\#F2F3F4) to Pale Orange Yellow (\#F3E5AB), flat to sligtly raised in the center, finely to coarsely granular (velvety to cottony in some strains), edge difuse (irregular or submerged in some strains), Light Orange Yellow (\#F3E5AB) to Vivid Yellow (\#F3C300), frequently Deep Reddish Brown (\#882D17) in the centre. Colonies at 30 ${ }^{\circ} \mathrm{C}$ in $7 \mathrm{~d}$ : SAB $25-35 \mathrm{~mm}$ diam $(\varnothing=31 \mathrm{~mm})$; MEA $39-48 \mathrm{~mm}$ diam $(\varnothing=39 \mathrm{~mm})$; PDA 35-42 mm diam $(\varnothing=36 \mathrm{~mm})$. Colonies at $37^{\circ} \mathrm{C}$ in $7 \mathrm{~d}$ : SAB $25-40 \mathrm{~mm}$ diam $(\varnothing=34 \mathrm{~mm})$; MEA $30-37 \mathrm{~mm}$ diam $(\varnothing=32 \mathrm{~mm})$; PDA $32-34 \mathrm{~mm}$ diam $(\varnothing=33 \mathrm{~mm})$. 
2 Specimens examined. NEW ZEALAND, hedgehog (Erinaceus europaeus), M.J. Marples (ex-holotype culture CBS 511.73 = ATCC 28443 = IMI 101051 = NCPF 375). THE NETHERLANDS, Delft (Diagnostic Center SSDZ), arm skin, human, 1979 (CBS 344.79). UNITED KINGDOM, Bristol (General Hospital Bristol), human, 1972 (IHEM 19619 = RV 28925); ibid., culture IHEM 19621 = RV 28927.

6

7

Distribution and ecology — Trichophyton erinacei is a zoophilic species that is common in wild-living and pet hedgehogs worldwide. The pathogen was originally described in the European hedgehog (Erinaceus europaeus), occurring naturally in the UK and Northern and Western Europe; it has also been imported to New Zealand and Japan Japan (Smith and Marples 1964, Morris and English 1969, Takahashi et al. 2003). The African wild-living four-toed hedgehog (Atelerix albiventris) is another host of T. erinacei. The prevalence of the pathogen is high in both wild-living and pet hedgehogs, resulting in a significant increase in human infections due to T. erinacei, especially those contracted from pet hedgehogs, in recent years (Abarca et al. 2017, Hubka et al. 2018c, Kargl et al. 2018). The presentation in hedgehog range from asymptomatic infection (Fig. 22) to extensive involvement of the body surface. The infection is predominantly located on the head and usually spread slowly (Morris and English 1973, Takahashi et al. 2002, Schauder et al. 2007). In human, extremities are affected in cca $70-80 \%$ of reported cases (Fig. 22), although tinea corporis, barbae, faciei (Fig. 22), capitis and onychomycosis have been also reported (English et al. 1962, Piérard-Franchimont et al. 2008, Concha et al. 2012).

Notes - The morphology of T. erinacei resembles that of T. africanum and T. mentagrophytes. Compared to T. mentagrophytes, with a dark colony reverse colour, the colony reverse colour of $T$. erinacei is pale. These species also differ in the production of spiral hyphae, which are absent in $T$. erinace $i$, and by the general shape of microconidia, which are mostly globose or subglobose in $T$. mentagrophytes. The species is strongly associated with hedgehogs, and identification is thus only difficult when isolated from infected humans with incomplete anamnestic data. The closely related taxa T. eriotrephon and T. verrucosum are easily distinguishable from T. erinacei by their slower growth rates (Figure 11) and relatively poor sporulation (sporulation usually absent in T. verrucosum). Additionally, T. eriotrephon can be differentiated from T. erinacei by the production of an intense reddish-brown pigment and microconidia with variable shapes. Only the MAT1-1-2 idiomorph was detected among the T. erinacei isolates examined here.

Trichophyton eriotrephon Papegaay, Ned. Tijdschr. Geneesk. 69: 885. 1925 — Figure 23 
Vegetative hyphae smooth, septate, hyaline, 1.4-3.2 $\mu \mathrm{m}$ diam (mean $\pm \mathrm{sd}$ : $2.2 \pm 1.0$ ). Well-differentiated conidiophores rare, usually only poorly differentiated from vegetative hyphae, lateral branches arise in a right-angle to the fertile hyphae, fertile hyphae frequently disintegrate into propagules (intercalary conidia and microconidia). Microconidia abundant, sessile, formed terminally or laterally on fertile hyphae, or on lateral branches, occasionally in short chains, variable in shape, mostly ovoid or pyriform, occasionally barrel-shaped, limoniform or irregular, 3.3-6.6 (4.6 \pm 0.85$) \times 2.1-3.7(3.4 \pm 0.41) \mu \mathrm{m}$ diam; intercalary conidia common, occasionally arranged in chains, barrel-shaped or irregular. Macroconidia absent. Spiral hyphae absent. Chlamydospores common. Sexual morph unknown.

Culture characteristics - (Colonies in $7 \mathrm{~d}$ at $\left.25^{\circ} \mathrm{C}\right)$ Colonies on SAB $17-25 \mathrm{~mm}$ diam $(\varnothing=21 \mathrm{~mm})$, White (\#F5F5F0) or Light Yellowish Brown (\#E3D6A1), flat with radially wrinkled centre, velvety to delicately granular, edge entire, reverse Deep Reddish Brown (\#882D17), diffuse pigment Strong Reddish Brown (\#6E2615) produced into the medium (less intense in IHEM 24340). Colonies on MEA 17-25 mm diam ( $\varnothing=21 \mathrm{~mm}$ ), White (\#F5F5F0) to Pale Yellow (\#C2B280) in the centre, flat with or without radially wrinkled centre, velvety to delicately granular, edge submerged and filliform, reverse Vivid Red (\#841B2D) to Deep Reddish Brown (\#882D17) (yellow reverse in IHEM 24340). Colonies on PDA 17-24 mm diam ( $\varnothing=22 \mathrm{~mm}$ ), White (\#F5F5F0) to Light Yellowish Brown (\#E3D6A1) in the centre, flat or umbonate, with raised centre (radially wrinkled in CBS 220.25), velvety or downy, edge submerged to filliform, reverse Vivid Orange (\#F38400) to Strong Yellowish Brown (\#80461B) in the centre. Colonies at $30^{\circ} \mathrm{C}$ in $7 \mathrm{~d}$ : SAB 22-32 mm diam $(\varnothing=27 \mathrm{~mm})$; MEA 28-31 mm diam $(\varnothing=31$ $\mathrm{mm})$; PDA $22-25 \mathrm{~mm}$ diam $(\varnothing=24 \mathrm{~mm})$. Colonies at $37^{\circ} \mathrm{C}$ in $7 \mathrm{~d}$ : SAB $0-3 \mathrm{~mm} \operatorname{diam}(\varnothing=1 \mathrm{~mm})$; no growth on MEA and PDA.

Specimens examined. THE NEDERLANDS, human dermatophytosis, 1925, J. Papegaay (ex-type culture CBS 220.25). BELGIUM, Marke, dog skin and hair (Jack Russell terrier), 2010 (IHEM 24340).

Distribution and ecology — Insufficient data are available regarding the distribution of T. eriotrephon, which is known from four cases of dermatophytosis in humans (tinea corporis, Netherlands; tinea manuum and tinea faciei, Iran; tinea barbae, France) (Papegaay 1925, Rezaei-Matehkolaei et al. 2013, Sabou et al. 2018) and a dog (isolate IHEM 24340 from Belgium). ). It is assumed that T. eriotrephon is a zoophilic species based on its phylogenetic relationships with other zoophilic species and he clinical manifestations of known infections in humans.

Notes - The morphology of $T$. eriotrephon only slightly resembles species from the $T$. benhamiae clade in its red-brown colony reverse colour. The conidiophores of T. eriotrephon are mostly loose and poorly branched compared to those of zoophilic species from the $T$. benhamiae clade, with grape-like conidiophores. Other typical characteristics include the production of a diffuse red-brown pigment on 
$\mathrm{SAB}$, microconidia with variable shapes and the absence of macroconidia. These characteristics, together with the absence of or restricted growth at $37{ }^{\circ} \mathrm{C}$, differentiate $T$. eriotrephon from all other species of the T. benhamiae complex. The MAT1-1-1 idiomorph of the mating type gene was detected in both T. eriotrephon isolates examined here.

Trichophyton verrucosum E. Bodin, Les champignons parasites de l'homme: 121.1902 — Figure 24

Vegetative hyphae smooth, septate, frequently inflated, hyaline, 1-2.5 $\mu \mathrm{m}$ diam (mean \pm sd: $1.7 \pm$ 1.16). Conidiophores rare, poorly differentiated from vegetative hyphae, unbranched or sparsely branched, conidia sessile on hyphae or born on short lateral branches. Microconidia absent or rare, clavate, 3-6 (4.5 \pm 0.7$) \times 1.9-3.5(2.9 \pm 0.45) \mu \mathrm{m}$. Macroconidia absent or rare, smooth-walled, clavate or fusiform with rounded apex and truncate end, usually consisting of 1-4 cells (median $=2$ ), 16-50 $\times$ 4-8 $\mu \mathrm{m}$. Chlamydospores abundant and frequently in the form of chains. Spiral hyphae absent. Sexual morph unknown.

Culture characteristics - (Colonies in $7 \mathrm{~d}$ at $\left.25{ }^{\circ} \mathrm{C}\right)$ Colonies on SAB $18-22 \mathrm{~mm}$ diam $(\varnothing=20 \mathrm{~mm})$, White (\#F5F5F0) to Pale Orange Yellow (\#FFF587) or Light Orange Yellow (\#FAD6A5), flat, raised and furrowed, or cerebriform, velvety to sligtly powdered, edge entire, lobate, or submerse, reverse Light Orange Yellow (\#F8DE7E) to Deep Orange Yellow (\#C9AE5D), dark brown in some strains. Colonies on MEA 5-18 mm diam ( $\varnothing=13 \mathrm{~mm}$ ), White (\#F5F5F0) to Pale Orange Yellow (\#F3E5AB), raised in the centre, frequently wrinkled, velvety or waxy, edge entire, lobate, or submerse, reverse Light Orange Yellow (\#F8DE7E) to Vivid Orange Yellow (\#F6A600), dark brown in some strains. Colonies on PDA 8-18 mm diam ( $\varnothing=14 \mathrm{~mm}$ ), White (\#F5F5F0) to Pale Orange Yellow (\#F3E5AB), flat or with raised centre, velvety or waxy, edge entire, lobate, or submerse, reverse Light Orange Yellow (\#F8DE7E), dark brown in some strains. Colonies at $30^{\circ} \mathrm{C}$ in $7 \mathrm{~d}$ : SAB $10-23 \mathrm{~mm}$ diam $(\varnothing=$ $18 \mathrm{~mm})$; MEA $8-10 \mathrm{~mm}$ diam $(\varnothing=9 \mathrm{~mm})$; PDA $9-10 \mathrm{~mm} \operatorname{diam}(\varnothing=9 \mathrm{~mm})$. Colonies at $37{ }^{\circ} \mathrm{C}$ in $7 \mathrm{~d}$ : SAB 9-15 mm diam ( $\varnothing=10 \mathrm{~mm})$; MEA $11-12 \mathrm{~mm}$ diam $(\varnothing=11 \mathrm{~mm})$; PDA $12-14 \mathrm{~mm} \operatorname{diam}(\varnothing=13$ $\mathrm{mm})$.

Specimens examined. CZECHIA, Pardubice, dermatophytosis in 21-year-old woman (contact with cattle), 2011, K. Mencl (CCF 4612). CZECHIA, Hlinsko, dermatophytosis in 58-year-old woman (contact with cattle), 2011, K. Mencl (CCF 4613). CZECHIA, Tábor, dermatophytosis in 38-year-old woman (contact with cattle), 2014, N. Mallátová (CCF 4889).

Distribution and ecology - Trichopyhton verrusocum is a zoophilic species typically found in cattle and other ruminants (Fig. 22), but it can easily spread to humans and animals, including horses, donkeys, 
camels, rabbits, dogs, cats, pigs, and even birds (Georg 1960, Dvořák et al. 1965, Ali-Shtayeh et al. 1988, Khosravi and Mahmoudi 2003, Chermette et al. 2008). The species is distributed worldwide, but the incidence of infections in cattle and man has been decreased in many regions by specific preventive measures, especially by vaccination programmes or changes in agricultural systems, such as reduction of the number of cattle in breeding units, and infections in humans have decreased proportionally (Seebacher et al. 2008, Lund et al. 2014). Human patients usually develop aggressive inflammatory skin lesions usually located on extremities and head (Fig. 22), which may be accompanied by constitutional symptoms, such as fever and lymphadenopathy (Silver et al. 2008, Courtellemont et al. 2017). Tinea barbae and capitis are relatively common clinical forms which can result in irreversible scarring and alopecia.

Notes - The morphology of T. verrucosum resembles T. bullosum and T. concetricum. For distinguishig characters see T. bullosum description. Only MAT1-2-1 idiomorph was detected in all strains examined here and in all strains analyzed by other reserchers (Kano et al. 2014, Kosanke et al. 2018).

\section{Trichophyton bullosum clade}

Trichophyton africanum Cmokova \& Hubka, sp. nov. — MycoBank XXXX; Figure 25

Etymology. Refers to the origin of the ex-type strain.

Vegetative hyphae smooth, septate, hyaline, $1-4 \mu \mathrm{m}$ diam (mean $\pm \mathrm{sd}: 2.2 \pm 0.5$ ). Conidiophores poorly differentiated from vegetative hyphae, unbranched or sparsely branched, conidia sessile on lateral or terminal branches. Microconidia abundant, pyriform to clavate, $2.5-5(4 \pm 0.5) \times 1.9-2.9(2.4 \pm 0.3)$ $\mu \mathrm{m}$. Macroconidia abundant, cigar-shaped, 14-80.5 (64.2 \pm 14.4$) \times 6-11(8.2 \pm 1.2) \mu \mathrm{m}$, consisting of 3-9(-13) cells (median $=6$ ). Chlamydospores present. Spiral hyphae rare or absent. Sexual morph unknown.

Culture characteristics - (Colonies in 7 d at $\left.25^{\circ} \mathrm{C}\right)$. Colonies on SAB $30-32 \mathrm{~mm}$ diam $(\varnothing=31 \mathrm{~mm})$, White (\#F2F3F4) to Pale Yellow Green (\#F2F3E5), granular, slightly raised in the center, margin diffuse, reverse Light Orange Yellow (\#FBC97F) in the marginal part, Strong Orange (\#ED872D) in the center. Colonies on MEA 28-35 mm diam $(\varnothing=30 \mathrm{~mm})$, White (\#F2F3F4), granular, flat, margin entire, reverse Light Orange Yellow (\#FBC97F). Colonies on PDA 27-28 mm diam ( $\varnothing=28 \mathrm{~mm}$ ), White (\#F2F3F4) to Pale Yellow Green (\#F2F3E5), granular to floccose, slightly raised in the center, margin entire, reverse Pale Yellow (\#F3E5AB) to Pale Orange Yellow (\#FAD6A5) in the marginal part, Dark Orange Yellow (\#BE8A3D) in the center. Colonies at $30{ }^{\circ} \mathrm{C}$ in $7 \mathrm{~d}$ : SAB $40-45 \mathrm{~mm}$ diam $(\varnothing=43 \mathrm{~mm})$; 
MEA 35-45 mm diam $(\varnothing=39 \mathrm{~mm})$; PDA $35-40 \mathrm{~mm}$ diam $(\varnothing=36 \mathrm{~mm})$. Colonies at $37^{\circ} \mathrm{C}$ in $7 \mathrm{~d}$ : SAB $21-24 \mathrm{~mm}$ diam $(\varnothing=24 \mathrm{~mm})$; MEA $20-29 \mathrm{~mm}$ diam ( $\varnothing=23 \mathrm{~mm})$; PDA 20-22 $\mathrm{mm}$ diam $(\varnothing=21 \mathrm{~mm})$.

3

Specimens examined. MozAMBIQUE, human, 1969, M.J. Campos-Magalhaes (PRM 944418, holotype, dried culture; culture ex-type IHEM 4032 = ATCC 28064 = RV 25293 = CM 3440). BELGIUM, Bruges, human fingernail, 1978 (IHEM 19628 = RV 40614). SOUTH AFRICA, human skin, 1971, K. Scott (IHEM $4033=$ ATCC $28065=$ CBS 808.72 = CECT $2895=$ NCPF $456=$ RV 27926).

Distribution and ecology - All three currently known strains are of human origin, but the low number of isolates does not allow us to draw conclusions about their ecology. The species probably occurs mainly in Africa.

Notes - Some aspects of the morphology of T. africanum resemble those of zoophilic species from the T. benhamiae clade, T. erinacei and T. mentagrophytes sensu de Hoog et al. (2017). Trichophyton africanum shows a faint apricot colony reverse colour, differing from the intense yellow or red/brown pigments typical of $T$. benhamiae clade species and T. mentagrophytes. The conidiophores of $T$. africanum are unbranched or sparsely branched; when branched, the resulting conidiophores have usually only few and relatively long lateral branches and are less compact than those of $T$. benhamiae var. luteum, T. europaeum and T. japonicum (pyramidal/grape-like with many short lateral branches). Trichophyton africanum has conidia of similar lengths to those of $T$. benhamiae var. benhamiae and in average longer than those of the remaining species from the $T$. benhamiae clade. The differentiation of this species from T. erinacei on the basis of morphology may be difficult, but T. erinacei is very strongly associated with hedgehogs. The most closely related species, $T$. bullosum, can be easily distinguished by its very slow growth, poor or absent sporulation, and abundant production of chlamydospores. The ratio of MAT1-1-1 and MAT1-2-1 strains in T. africanum was 2:1.

Trichophyton bullosum Lebasque, Les Champignons des Teignes du Cheval et des Bovidés: 53. 1933 - Figure 26

Vegetative hyphae smooth, septate, inflated, often branched andwith knob-like terminations, hyaline 1.5-4 $\mu \mathrm{m}$ diam (mean $\pm \mathrm{sd} ; 2.7 \pm 0.7$ ). Chlamydospores abundant, spherical, oval or irregular, occasionally in chains, $4-9(-20) \mu \mathrm{m}$ in diam. Microconidia and macroconidia not observed in the isolates examined in this study, but they were observed by Lebasque (1933) under specific conditions. Spiral hyphae absent.

Culture characteristics - (Colonies in $7 \mathrm{~d}$ at $\left.25^{\circ} \mathrm{C}\right)$ Colonies on SAB $11-12 \mathrm{~mm}$ diam $(\varnothing=12 \mathrm{~mm})$, White (\#F2F3F4) to Pale Yellowish Pink (\#ECD5C5) or Pale Orange Yellow (\#FAD6A5), umbonate, 
radially furrowed, membranous or slightly velvety, edge submerged or filiform, reverse Light Yellow (\#F8DE7E). Colonies on MEA 8-12 mm diam $(\varnothing=10 \mathrm{~mm})$, White (\#F2F3F4), Pale Yellow (\#F3E5AB) or Vivid Orange Yellow (\#F6A600), flat with raised and cerebriform center, membranous, edge entire or submerged with dendritic growth, reverse Light Yellow (\#F8DE7E). Colonies on PDA 7-9 mm v diam ( $\varnothing=8 \mathrm{~mm}$ ), White (\#F2F3F4), Pale Yellowish Pink (\#ECD5C5) or Pale Orange Yellow (\#FAD6A5), circular, flat, umbonate, membranous, edge entire, reverse Light Yellow (\#F8DE7E). Colonies at $30^{\circ} \mathrm{C}$ in $7 \mathrm{~d}$ : SAB $11-14 \mathrm{~mm}$ diam $(\varnothing=13 \mathrm{~mm})$; MEA $11-12 \mathrm{~mm}$ diam $(\varnothing=11 \mathrm{~mm})$; PDA $12-13 \mathrm{~mm}$ diam $(\varnothing=12 \mathrm{~mm})$. Colonies at $37{ }^{\circ} \mathrm{C}$ in $7 \mathrm{~d}$ : SAB $8-10 \mathrm{~mm}$ diam $(\varnothing=9 \mathrm{~mm})$; MEA 9-10 $\mathrm{mm} \operatorname{diam}(\varnothing=10 \mathrm{~mm})$; PDA 9-10 $\mathrm{mm} \operatorname{diam}(\varnothing=9 \mathrm{~mm})$.

Specimens examined. FRANCE, horse, J. Lebasque (ex-type culture, CBS $363.35=$ LP 770). CZECHIA, skin lesions in horse, 2013, P. Lysková (CCF 4831). EGYPT, near Cairo, skin lesion in donkey (Equus asinus), 2015, A. Peano (CCF 5730).

Distribution and ecology - Trichophyton bullosum is a zoophilic species known from infections in donkeys and horses (Fig. 27). It is distributed in Europe, North Africa and the Middle East (Lebasque 1933, Sitterle et al. 2012, Lysková et al. 2015, Sabou et al. 2018).

Notes - Due to its slow grow rate, T. bullosum strongly resembles $T$. verrucosum and $T$. concetricum. These species either do not sporulate or sporulate poorly (especially on sugar-rich media such as SAB) but produce abundant chlamydospores, frequently in the form of chains. All mentioned species are relatively strongly associated with their hosts and/or with a typical clinical manifestation (cattle ringworm caused by $T$. verrucosum; dermatophytosis caused by $T$. bullosum in horses and donkeys; tinea imbricata caused by $T$. concentricum in humans). Therefore, detailed anamnestic data can facilitate their identification. Molecular genetic methods may be necessary to verify the identification of some isolates. For the differentiation of $T$. bullosum from the most closely related species, $T$. africanum, see the description of T. africanum. Only the MAT1-1-1 idiomorph was detected among the T. bullosum isolates examined here.

\section{DISCUSSION}

\section{Species delimitation issues in Trichophyton}

Species delimitation in dermatophytes is based on a polyphasic approach (Gräser et al. 2008) combining ecological (distribution, host range) and clinical data, the analysis of DNA sequence data, the macroand micromorphological examination of cultures, physiological and biochemical tests and mating tests. However, the application of the individual components of this concept is limited in many species complexes due to specific problems. As a result, the "polyphasic" approach is commonly applied in a restricted form in practice. 
Phenotypic criteria are usually relatively effective in routine diagnostics for major dermatophyte species or species complexes. However, as in other fungi, we have found similarities between species or morphotypes across unrelated dermatophytes, resulting in misdiagnosis in practice (Summerbell 2011, Lysková et al. 2015, Uhrlaß et al. 2018). There is also considerable intraspecific phenotypic variability in other species or species complexes that is not correlated with molecular

6

7 taxonomy (Heidemann et al. 2010, Su et al. 2019, Kandemir et al. 2020). Moreover, the success rate of phenotypic identification frequently depends on the age of isolates because of the rapid degeneration of important portions of cultures (de Hoog et al. 2017). Consequently, it can be difficult to maintain and reproduce phenotypic characters over decades for the purposes of taxonomic studies.

The high level of clonality in many primary pathogenic dermatophytes with a presumed recent origin is also associated with an extremely low level of genetic intraspecific variability. Consequently, there is a lack of sufficiently variable DNA sequence markers for the differentiation of some species and, therefore, ambiguities in the definition of their boundaries (de Hoog et al. 2017). Phenomena such as incomplete lineage sorting or occasional hybridization and introgression may further complicate the species delimitation of evolutionarily recently diverged species with semi-permeable reproductive barriers (Taylor et al. 2015, Steenkamp et al. 2018, Matute and Sepúlveda 2019). The divergence between these young species may be hidden when using some classical protein-coding phylogenetic markers. Neutrally evolving or noncoding DNA regions, such as microsatellites, introns and intergenic spacers, which accumulate mutations more rapidly, were shown to reveal the evolutionary trajectories of primary pathogenic dermatophytes with higher success (Gräser et al. 2008, Mochizuki et al. 2017, Hubka et al. 2018c).

The specific problems in species delimitation in Trichophyton can be demonstrated by the example of the T. mentagrophytes and Trichophyton rubrum complexes. It was generally assumed that the differentiation of zoophilic T. equinum (main host = horse) from closely related anthropophilic $T$. tonsurans would be possible based on the ecological preferences, nutritional requirements, and MAT gene idiomorphs of the fungi (Woodgyer 2004, Summerbell et al. 2007). Kandemir et al. (2020) examined 67 isolates and found that none of the five selected phylogenetic markers were able to unambiguously separate these species (probably due to incomplete lineage sorting) according to differences in their MAT genes, ecology and nicotinic acid requirements. It is postulated that these species evolved very recently and that the speciation process might not yet be complete (Kandemir et al. 2020). Another taxonomically problematic species pair is T. mentagrophytes/T. interdigitale. According to the traditional concept promoted by de Hoog et al. (2017), T. mentagrophytes is a zoophilic species in which both MAT idiomorphs are present in the population, resulting in relatively high intraspecific genetic variability. By contrast, anthropophilic T. interdigitale is a clonal lineage (consisting only of the MAT1-1-1 idiomorph) that is almost exclusively associated with onychomycosis and tinea pedis. Although the correlation between the genotype and the clinical manifestation or source of isolates has been repeatedly demonstrated, the correlation between ITS genotype and phenotype is 
relatively poor (Heidemann et al. 2010, Pchelin et al. 2016, Dhib et al. 2017). Currently, the molecular diagnosis of these species is mostly based on several unique sites in the ITS region, and phylogenies usually resolve T. mentagrophytes as para- or polyphyletic with T. interdigitale (Heidemann et al. 2010, Nenoff et al. 2019, Pchelin et al. 2019, Singh et al. 2019, Taghipour et al. 2019, Hainsworth et al. 2020). Both species names remain in use, due to the epidemiological consequences associated with different sources of infections in particular. The laboratory diagnosis of T. mentagrophytes and T. interdigitale and that of T. equinum and T. tonsurans are further complicated by inaccurate or even impossible species differentiation using MALDI-TOF MS (Nenoff et al. 2013, da Cunha et al. 2018, Dukik et al. 2018, Suh et al. 2018, Hedayati et al. 2019).

Very similar species delimitation issues complicate the taxonomy of the anthropophilic $T$. rubrum complex, encompassing clonal lineages showing differences in their distribution and the clinical manifestation of associated infections (Gräser et al. 2000, de Hoog et al. 2017). The majority of molecular studies relying on the variability in the ITS region and microsatellite markers have revealed some support for 2-4 lineages (i.e., T. rubrum, T. violaceum and/or T. soudanense and/or T. yaoundei), but the number of species and their boundaries are still under debate (Gräser et al. 2007, Su et al. 2019, Packeu et al. 2020). MALDI-TOF MS showed promising results in the differentiation of these species/lineages (Packeu et al. 2020).

Detailed genomic, epigenetic and multigene phylogenetic studies on a large number of samples can resolve delimitation issues between these species in the future (Zhan et al. 2018, Pchelin et al. 2019, Singh et al. 2019). SNP detection by whole-genome sequence typing can be used to infer the genetic relatedness of Trichophyton isolates. This approach will ultimately become one of the methods of choice in the future with decreasing costs (Hadrich and Ranque 2015). Currently, the sequencing of ITS rDNA and population genetic markers such as microsatellites (Kaszubiak et al. 2004, Gräser et al. 2007, Pasquetti et al. 2013) or mixed-marker approaches (Abdel-Rahman et al. 2010) offers higher discriminatory power in the species differentiation of primary pathogenic dermatophytes compared to MLST approaches based on the currently available loci.

\section{Disentangling the taxonomy of the $T$. benhamiae complex based on a polyphasic approach}

In this study, we encountered similar problems to those mentioned in the previous chapter in the $T$. benhamiae complex. However, a polyphasic approach combining independent molecular genetic markers (four DNA loci and 10 microsatellite loci) with phenotypic features and ecological data helped to overcome the majority of obstacles to species delimitation.

We showed that isolates that were designated in the past as the European-American race of $T$. benhamiae harbour five taxa (three species and two varieties). The strains with the so-called white phenotype do not represent monophyletic entities and correspond to T. benhamiae var. benhamiae, $T$. japonicum and T. europaeum, while the yellow phenotype strains correspond to T. benhamiae var. luteum. Isolates of the African race of $T$. benhamiae referred to as $T$. africanum herein are 
phylogenetically distant and are most closely related to T. bullosum. The main characteristics of these species and features that are useful for their identification are schematically summarized in Figure 28.

None of the four sequence markers alone was able to unequivocally differentiate all species within the T. benhamiae complex and provide accurate identification in $100 \%$ of cases. The ITS region contained a diagnostic position for all nine species, but the differentiation of $T$. europaeum and $T$. japonicum relied on a single substitution. In addition, the identification of isolate IHEM 25139, with a probable hybrid origin, failed as described above. The gapdh gene was useful for differentiation between T. europaeum and T. japonicum, but some pairs of sister species shared identical sequences (i.e., T. benhamiae and T. concentricum, $T$. verrucosum and T. eriotrephon). The tefl- $\alpha$ gene differentiated all species except for T. europaeum and T. japonicum. The tubb gene presented the least discriminatory power and failed to differentiate species within the $T$. benhamiae clade but could be used for species identification in the T. erinacei and T. bullosum clades. Insufficient discriminatory power of the tubb gene has been reported in many other Trichophyton species (Suh et al. 2018, Kandemir et al. 2020, Packeu et al. 2020). The unique substitutions observed within the DNA loci of the T. benhamiae clade species will be the basis for reliable species identification in practice. The taxonomic significance of these unique sites is unambiguous, as they correspond to independent microsatellite markers and phenotypic and ecological data, indicating the reproductive isolation of recognized taxa.

While sequence markers were shown to be useful for the diagnosis of $T$. benhamiae complex species, they were not able to distinguish the two varieties of $T$. benhamiae. The only intraspecific variation was a single substitution in the tefl- $\alpha$ gene. This substitution was able to differentiate all $T$. benhamiae var. luteum isolates from the majority of $T$. benhamiae var. benhamiae strains, with the exception of two isolates from cluster $\mathrm{C} 2$, probably due to incomplete lineage sorting between these recently diverged lineages. The results of other analyses clearly indicated that $T$. benhamiae var. luteum is an emerging entity that is distinct both qualitatively (at the population genetic level and according to phenotypic differences) and ecologically (showing different hosts and distributions). The differentiation of this taxon has clinical relevance, due to which we decided to reassign it as variety of the nearest recombining ancestor, $T$. benhamiae var. benhamiae. We chose this conservative approach rather than the proposals of a new species because of the impossibility of distinguishing this entity using available DNA sequence markers, as is the current standard in fungal taxonomy.

In contrast to DNA sequence data, population genetic analysis based on the newly developed microsatellite typing scheme clearly separated all species in the $T$. benhamiae clade (Figures 5-6), including T. benhamiae var. benhamiae and T. benhamiae var. luteum. Similarly, pilot MALDI-TOF MS analysis was able to identify specific peaks for all species and varieties in the $T$. benhamiae clade, suggesting that this increasingly popular method can be used for species identification in clinical practice, but the analysis of additional isolates will be needed to generate a more robust database and confirm our preliminary observations. 
Phenotypic and ecological data added another important piece to the taxonomic puzzle. Trichophyton benhamiae var. luteum can be identified by its slow growth on all media at all temperatures and its uniform phenotype (yellow reverse side of colonies and absence of macroconidia; all strains exhibit only mating type MAT1-1-1). The closely related T. benhamiae var. benhamiae is only found in the USA (mostly dogs) and exhibits strikingly different colonies with a brown to redbrown reverse side, macroconidium production and larger microconidia than T. benhamiae var. luteum. This variety shows the most rapid growth among the species from the $T$. benhamiae clade; isolates with both MAT gene idiomorphs were detected among the examined strains. Trichophyton europaeum is the second most common species from the T. benhamiae complex occurring in Europe and is responsible for human and guinea pig infections. While T. japonicum is currently responsible for the majority of human and animal (rabbits and guinea pigs) infections in Japan, it also occurs in Europe at low frequencies. Reliable differentiation of these species is only possible by molecular methods (Figure X). Trichophyton japonicum and T. europaeum differ strikingly in the distribution of mating type genes in their populations. Detailed distinguishing characteristics of particular species are listed in the Taxonomy section, and some important characteristics are summarized in Figure 28.

\section{Speciation through host switching and the extinction of opposite mating type partners}

The assessment of species boundaries via mating experiments (revealing biological compatibility) played an important role in the delimitation of many early species and the discovery of their sexual states. This approach based in principle on the biological species concept (BSC) is generally highly applicable in geophilic dermatophytes (Dawson and Gentles 1962, Stockdale 1964, Padhye and Carmichael 1972, Choi et al. 2012, Hubka et al. 2015a). By contrast, the results of biological compatibility assessment can considerably disagree with the concept of classical species of anthropophilic and zoophilic dermatophytes. These species are evolutionarily young, and their phylogenetic divergence preceded the development of reproductive barriers, as demonstrated by interspecific hybrid induction between various primary pathogenic Trichophyton species in vitro (Kawasaki et al. 2009, Anzawa et al. 2010, Kawasaki et al. 2010, Kawasaki 2011). However, it is highly unlikely that this kind of hybridization occurs naturally due to the different ecological niches of species, and the results of in vitro mating assays therefore cannot be extrapolated to a natural scenario. Additionally, the ratio of mating-type gene idiomorphs is usually extremely imbalanced or one idiomorph is missing in the majority of anthropo- and zoophilic dermatophytes (Metin and Heitman 2017, Kosanke et al. 2018). This fact further limits or even prevents the possibility of using BSCs in the delimitation of these species. A similar phenomenon was observed by our group in all species from the T. benhamiae complex (Figure 28), suggesting that the loss of opposite mating-type partners was an important driver of their evolution. The ancestors of many currently recognized pathogenic dermatophytes were likely sexually reproducing geophilic species and zoophilic species on free-living mammals (sexually reproducing, e.g., in soil surrounding burrows) with balanced ratios of opposite 
mating type individuals (Gräser et al. 2006, Summerbell 2011). Adaptation to a new host is probably a unique event in the evolution of many anthropo- and zoophilic dermatophytes, resulting in the extinction of one mating partner in the whole population of these species. Only some "clonal" offshoots of ancestral sexual dermatophytes probably maintain ongoing populations and follow independent evolutionary trajectories towards speciation (Gräser et al. 2006). Alternatively, the extinction of one MAT gene in a population of dermatophytes may be caused by the preferential spread of strain(s) exhibiting an advantageous combination of alleles associated with higher virulence/transmission potential. Such a successful genotype may be significantly dominant in conditions with almost exclusive asexual transmission and may displace other genotypes. Such a situation is very likely to lead to an imbalance in the MAT gene ratio or even the loss of one MAT gene in the population. The extinction of strains belonging to one mating type is, for instance, observed in some populations of $M$. canis (Sharma et al. 2007), and different levels of virulence linked with mating-type idiomorphs have been repeatedly documented in fungal pathogens (Yue et al. 1999, Chang et al. 2000, Cheema and Christians 2011)

In the T. benhamiae clade, clonal reproduction is the dominant mode of dissemination (Dg, $\mathrm{H}$, DW indices), and recombination is rare or absent in almost all populations according to the $\mathrm{I}_{\mathrm{A}}$. Despite the fact that only MAT1-2-1 idiomorph strains were present within $T$. europaeum strains, the null hypothesis of random mating was not rejected (Table S6, Figure 9), suggesting the existence of recent recombination events in this species. As T. japonicum and T. europaeum consist of a single mating type and no recent recombination or gene flow has occurred between them, they should be conceptualized as separate, albeit clonal species, despite potential in vitro interbreeding (Summerbell 2002, Gräser et al. 2006). The disruption of gene flow between T. benhamiae clade species was demonstrated by fixation indices ( $\mathrm{F}_{\text {ST }}$ or $\mathrm{G}_{\mathrm{ST}}$ ) (Table S5, Table S6).

In the T. benhamiae complex, there are at least two possible sexual ancestors of "clonal" species: T. benhamiae var. benhamiae and T. africanum, based on the presence of both MAT gene idiomorphs. While the ecology of T. africanum is poorly known, reservoirs of $T$. benhamiae var. benhamiae exist in free-living animals. It has been detected in the North American porcupine (Takahashi et al. 2008, Needle et al. 2019), but its host spectrum can be broader and may include members of family Canidae, as evidenced by repeated isolation from dogs (Ajello and Cheng 1967, Sieklucki et al. 2014) and patients who have come into contact with foxes (Tan et al. 2020). Due to close phylogenetic proximity, Trichophyton benhamiae var. benhamiae was very likely a common ancestor of at least some taxa in the T. benhamiae clade, especially anthropophilic T. concentricum (only MAT1-1-1) and zoophilic T. benhamiae var. luteum (only MAT1-1-1). The low genetic diversity within $T$. benhamiae var. luteum together with its recent origin (according to the DW index) may indicate a founder effect in the recent past. This may suggest that the origin of $T$. benhamiae var. luteum lies in North America and that one or a few strains were recently introduced to Europe. 
The only exception among the examined isolates was strain IHEM $25139(=$ RV $14387=$ ATCC $28061=$ CBS $806.72=$ IFM 54422), isolated in 1963 by M. Takashio from guinea pig in France. This strain, identified here as T. europaeum based on the gapdh gene, shared some microsatellite alleles with T. japonicum. It also presented the MAT1-1-1 idiomorph of the MAT gene, typical of T. japonicum or T. benhamiae var. benhamiae cluster C3, and an atypical ITS1 region sequence with six substitutions compared to other T. europaeum strains, some of which are at positions crucial for the differentiation of T. benhamiae clade species (Figure S2). It is possible that this strain originated from hybridization between T. europaeum and T. japonicum. The ecological niches of these species partially overlap, as they both occur in guinea pigs and some other animals that are frequently maintained together. In addition, the coinfection of guinea pigs with two species or morphotypes has been repeatedly documented (Kupsch et al. 2017, Bartosch et al. 2019). In such cases, the exchange of genetic information may likely occur not only through hybridization during saprophytic growth outside the host (possibly followed by introgressive hybridization) but also during coinfection of the same host through a parasexual cycle (anastomosis of hyphae, mitotic crossing-over and haploidization). Another strain with an ITS sequence identical to IHEM 25139 is IHEM 19622 (= RV 14389), which was not examined by our group (GenBank MK298816). These two strains with identical provenance were noted by Takashio to be atypical compared to other examined A. benhamiae isolates because of the less compact texture of their colonies (Takashio 1974). These strains represent unique material for studying natural hybridization in dermatophytes. Their origin and genomic arrangement remain to be elucidated by genomic studies. The absence of these genotypes among the more recently isolated strains examined here and by others (no additional occurrence in GenBank) suggests that they were short-lived and were replaced by more successful genotypes.

\section{Geographical distribution of T. benhamiae clade species}

To understand the global distribution of the newly reassigned species in the $T$. benhamiae clade, we analysed several hundred ITS rDNA sequences deposited in GenBank. The analysis enabled the identification of these records to the species level based on the species-specific substitutions in the ITS region. This fact further supported the feasibility of the novel taxonomic classification proposed here. The ecological data resulting from the analysis were used as a basis for mapping the distribution of $T$. benhamiae clade species (Figure 29; Table S10). The main limitations are the unavailability of epidemiological and DNA data from America, many Asian countries and Africa. As a result, the majority of analysed ITS sequences are from European countries and Japan, where dermatophyte research has a long tradition, and DNA-based identification is more commonly used. Additionally, it is not possible to distinguish two varieties of $T$. benhamiae based on the ITS region, but macro- and micromorphological characters described in some studies enable clear distinction of the varieties; the variety characteristics described below refer to such cases. 
In Europe, guinea pigs are hosts of all three pathogens, among which $T$. benhamiae var. luteum is the most prevalent, followed by T. europaeum and T. japonicum. The ITS-based identification of 30 T. benhamiae strains from guinea pigs from a single veterinary institution in Prague (Czech Republic) between 2014-2019 revealed a 24:4:2 ratio of these pathogens (Hubka and Prausová, unpubl. data). The corresponding ratio of these pathogens in human Czech patients is very similar, 27:5:1 (Hubka et al. 2014, Hubka et al. 2018b, Hubka et al. unpubl. data). In addition to guinea pigs, another important reservoir of $T$. japonicum are rabbits, while other animal hosts of $T$. benhamiae clade members seem to be much less important.

Based on current knowledge, it is clear that white-phenotype strains of T. benhamiae occurred in Europe before epidemic spread of T. benhamiae var. luteum. The oldest European white-phenotype strains representing T. japonicum are IHEM 4030 (collected before 1988 in Belgium) and IHEM 17701 (collected before 1997 in Spain). The oldest white-phenotype strains representing T. europaeum were collected before 1988 in Finland (Aho 1980) (Table S10) and more recently from Switzerland, in 2002 (IHEM 20159, IHEM 20161, IHEM 20162, IHEM 20163). The identity of other old white-phenotype strains reported in various European countries from the 1960s to 2000 (Figure 1) is unclear due to the unavailability of isolates and/or sequence data. Both T. japonicum and T. europaeum were subsequently detected in Japan and some other countries (Figure 29, Table S10). Outbreaks of infections caused by T. benhamiae var. luteum now seem to be limited to Europe, but an increasing number of infections can be expected in non-European countries due to its recent introduction to other continents (Hiruma et al. 2015, de Freitas et al. 2019).

Zoophilic T. benhamiae clade members have probably been brought into Japan with imported animals on several occasions and spread in Japan by the transportation of animals by breeders or pet shops, as suggested in a series of publications (Kano et al. 1998, Mochizuki et al. 2001, Takeda et al. 2012, Hiruma et al. 2015). The most prevalent species in Japan and South Korea (Jun et al. 2004, Lee et al. 2018, and pers. comm. with PL Sun) is T. japonicum. Other species are probably much less common: T. europaeum has been detected in guinea pig (unknown year of isolation) (Takeda et al. 2012), T. benhamiae var. benhamiae was imported to a Japanese zoo from Canada and the USA (in 2000 and 2002) with North American Porcupines (Takahashi et al. 2008), and T. benhamiae var. luteum was detected in 2012 in common degu (Hiruma et al. 2015).

In addition to Europe and Japan, T. benhamiae var. luteum was recently reported in Brazil (de Freitas et al. 2019, Santana et al. 2020). Trichophyton benhamiae var. benhamiae was confirmed only in North America in our study but was also recently reported in China (Tan et al. 2020).

Animal trade certainly plays an important role in the spread of zoonotic dermatophytes to new geographic areas. It also erases original geographic areas of a species distribution. Consequently, it is difficult to trace the origin of particular species. The current worldwide distribution and prevalence of infections caused by $T$. benhamiae clade members are poorly known due to insufficient overall surveillance of dermatophytosis supported by molecular-based identification. This problem pertains to 


\section{6}

7

both human and veterinary medicine. In addition, our knowledge of the ecology of these pathogens is mostly limited to domestic animals and pets, and little is known about potential wild-living hosts. Therefore, any hypothesis about the species origin is based on very incomplete data and needs to be refined by future research.

\section{Genotyping and surveillance of emerging pathogens in the T. benhamiae complex}

The emergence and rapid spread of $T$. benhamiae in Europe in the last decade and the recent detection of this species in many other countries has been one of the major public health events in the field of zoonotic superficial mycoses in recent years. This fact underscores the need for the One Health integrative approach and closer collaboration between the veterinary profession, dermatologists, epidemiologists and public health personnel (Nenoff et al. 2014, Hubka et al. 2018c). Infected and frequently asymptomatic animals may act as a recurrent source of infections in other animals and humans. Interdisciplinary cooperation is needed to establish effective preventive measures for the control of infections.

Genotyping techniques are often employed to gain insight into the dynamics of disease transmission, determine the source and routes of infections, confirm or rule out outbreaks, recognize virulent strains and regional and global changes in genotype patterns and evaluate the effectiveness of control measures (Ranjbar et al. 2014). Other common issues in dermatophytes concern the differentiation of relapse versus reinfection and the determination of whether the infection is caused by one or more strains and if genotypes differ in their clinical manifestation. Many methods have been developed for the subtyping of dermatophytes, but a significant number of them are now obsolete, and their utility is frequently limited due to poor reproducibility or unsatisfactory strain differentiation (Abdel-Rahman 2008, Mochizuki et al. 2017, Hubka et al. 2018c). MLST typing approaches have been widely applied to many fungal pathogens (Meyer et al. 2009, Debourgogne et al. 2012, Bernhardt et al. 2013, Maitte et al. 2013, Prakash et al. 2016), but no such typing scheme has been evaluated and developed for dermatophytes, and the currently available loci usually lack sufficient discriminatory power to study the population structure of Trichophyton and Microsporum species in detail. Microsatellite markers are still among the most effective tools available for the subtyping of dermatophytes. Typing schemes have been developed for a limited number of species, including only T. rubrum (Gräser et al. 2007), Nannizzia persicolor (Sharma et al. 2008) and M. canis (Sharma et al. 2007, Pasquetti et al. 2013).

Polymorphisms in T. benhamiae (American-European race) were previously investigated by the RFLP analysis of the NTS region, which produced 11 different patterns in 46 isolates; this method successfully confirmed laboratory-acquired infections as well as familial outbreaks transmitted from pets (Mochizuki et al. 2002, Takeda et al. 2012). In this study, we developed a microsatellite typing scheme consisting of ten variable markers. This new typing scheme is currently the most powerful tool for the subtyping of $T$. benhamiae clade species. It is easy to use and cost-effective due to its multiplex 
design. It is possible that the modified scheme can be used in other species in the T. benhamiae complex. Our preliminary data showed that at least 6 of 10 markers (CT21b, TAG16, TC20, TCA16, TC19, TC17a) are useful for the subtyping of another emerging pathogen, T. erinacei.

The establishment of global databases based on largely comparable data, such as that from microsatellites, SNPs and DNA sequences, is desirable. Such databases would enable us to understand the global epidemiology of dermatophytes and monitor changes in genotype spectra on a global scale. Although high-throughput sequencing facilities are now widely available and increasingly used even in the epidemiology of fungal infections, this option has not yet been exploited in dermatophytes.

The prevalence and spread of emerging pathogens from the $T$. benhamiae complex require close monitoring, particularly because infection rates in the principal hosts (guinea pigs, hedgehogs, and others) are high. The new taxonomic classification and microsatellite typing scheme proposed in this study will enable the monitoring of changes in the frequencies of individual species and genotypes. It will help to evaluate the results of preventive measures and interventions and is a basic prerequisite for the preparation of epidemiological studies.

\section{ACKNOWLEDGEMENTS}

We are very grateful to Jan Karhan and Lukáš Vít Rýdl for the concept of data visualization and help with graphical adjustments of analysis outputs. This research was supported by the Charles University Grant Agency (GAUK 600217) and Czech Ministry of Health (grant AZV 17-31269A). Contribution of Vit Hubka was supported by the projects BIOCEV (CZ.1.05/1.1.00/02.0109) provided by the Ministry of Education, Youth and Sports of the Czech Republic and ERDF, and the Charles University Research Centre program no. 204069. We thank Milada Chudíčkova, Petra Seifertová and Adéla Kovaříčková for their invaluable assistance in the laboratory and Peter Mikula for research support. We thank Jiřina Stará, Magdalena Skořepová, Stanislava Dobiášová and Jana Hanzlíčková for providing some of the strains used in this study. The research reported in this publication was part of the longterm goals of the ISHAM working group Onygenales.

\section{REFERENCES}

Abarca M, Castellá G, Martorell J, Cabañes F. 2017. Trichophyton erinacei in pet hedgehogs in Spain: occurrence and revision of its taxonomic status. Medical Mycology 55: 164-172. Abdel-Rahman SM. 2008. Strain differentiation of dermatophytes. Mycopathologia 166: 319-333. Abdel-Rahman SM, Sugita T, González GM, et al. 2010. Divergence among an international population of Trichophyton tonsurans isolates. Mycopathologia 169: 1-13. 
Agapow PM, Burt A. 2001. Indices of multilocus linkage disequilibrium. Molecular Ecology Notes 1: 101-102.

Agnetti F, Righi C, Scoccia E, et al. 2014. Trichophyton verrucosum infection in cattle farms of Umbria (Central Italy) and transmission to humans. Mycoses 57: 400-405.

Ahdy AM, Sayed-Ahmed MZ, Younis EE, Baraka HN, El-khodery SA. 2016. Prevalence and potential risk factors of dermatophytosis in Arabian horses in Egypt. Journal of Equine Veterinary Science 37: 71-76.

Aho R. 1980. Pathogenic dermatophytes recovered from the hair of domestic animals in Finland between 1977 and 1980. Suomen Elainlaakarilehti 86: 487-506.

Ajello L, Cheng S-L. 1967. The perfect state of Trichophyton mentagrophytes. Sabouraudia 5: 230234.

Ali-Shtayeh M, Arda H, Hassouna M, Shaheen S. 1988. Keratinophilic fungi on the hair of cows, donkeys, rabbits, cats, and dogs from the West Bank of Jordan. Mycopathologia 104: 109121.

Anzawa K, Kawasaki M, Mochizuki T, Ishizaki H. 2010. Successful mating of Trichophyton rubrum with Arthroderma simii. Medical Mycology 48: 629-634.

Atlas RM. 2010. Handbook of Microbiological Media. CRC Press, Boca Raton, USA.

Bartosch T, Frank A, Günther C, et al. 2019. Trichophyton benhamiae and T. mentagrophytes target guinea pigs in a mixed small animal stock. Medical Mycology Case Reports 23: 37-42.

Benedict K, Jackson BR, Chiller T, Beer KD. 2018. Estimation of direct healthcare costs of fungal diseases in the United States. Clinical Infectious Diseases 68: 1791-1797.

Bernhardt A, Sedlacek L, Wagner S, et al. 2013. Multilocus sequence typing of Scedosporium apiospermum and Pseudallescheria boydii isolates from cystic fibrosis patients. Journal of Cystic Fibrosis 12: 592-598.

Bond R. 2010. Superficial veterinary mycoses. Clinics in Dermatology 28: 226-236.

Bonifaz A, Archer-Dubon C, Saúl A. 2004. Tinea imbricata or Tokelau. International Journal of Dermatology 43: 506-510.

Bonifaz A, Vazquez-Gonzalez D. 2011. Tinea imbricata in the Americas. Current Opinion in Infectious Diseases 24: 106-111.

Borman AM, Campbell CK, Fraser M, Johnson EM. 2007. Analysis of the dermatophyte species isolated in the British Isles between 1980 and 2005 and review of worldwide dermatophyte trends over the last three decades. Medical Mycology 45: 131-141.

Brasch J, Beck-Jendroschek V, Voss K, Uhrlaß S, Nenoff P. 2016. Arthroderma benhamiae strains in Germany. Morphological and physiological characteristics of the anamorphs. Hautarzt 67: 700-705. 
Burt A, Carter DA, Koenig GL, White TJ, Taylor JW. 1996. Molecular markers reveal cryptic sex in the human pathogen Coccidioides immitis. Proceedings of the National Academy of Sciences 93: 770-773.

Cafarchia C, Camarda A, Coccioli C, et al. 2010. Epidemiology and risk factors for dermatophytoses in rabbit farms. Medical Mycology 48: 975-980.

Clement M, Posada D, Crandall KA. 2000. TCS: a computer program to estimate gene genealogies. Molecular Ecology 9: 1657-1659.

Concha M, Nicklas C, Balcells E, et al. 2012. The first case of tinea faciei caused by Trichophyton mentagrophytes var. erinacei isolated in Chile. International Journal of Dermatology 51:283285.

Contet-Audonneau N, Leyer C. 2010. Émergence d'un dermatophyte transmis par le cochon d'Inde et proche de Trichophyton mentagrophytes var. erinacei: T. mentagrophytes var. porcellae. Journal de Mycologie Medicale 20: 321-325.

Courtellemont L, Chevrier S, Degeilh B, et al. 2017. Epidemiology of Trichophyton verrucosum infection in Rennes University Hospital, France: A 12-year retrospective study. Medical Mycology 55: 720-724.

Čmoková A. 2015. Molecular typization of isolates from Arthroderma benhamiae complex, a zoonotic agent of epidemic dermatophytosis in Europe. Master Thesis, Department of Botany, Charles University, Prague, Czech Republic.

da Cunha KC, Riat A, Normand AC, et al. 2018. Fast identification of dermatophytes by MALDITOF/MS using direct transfer of fungal cells on ground steel target plates. Mycoses 61: 691697.

Dawson CO, Gentles J. 1962. The perfect states of Keratinomyces ajelloi van-Breuseghem, Trichophyton terrestre Durie \& Frey and Microsporum nanum Fuentes. Sabouraudia 1: 4957.

de Freitas RS, de Freitas THP, Siqueira LPM, Gimenes VMF, Benard G. 2019. First report of tinea corporis caused by Arthroderma benhamiae in Brazil. Brazilian Journal of Microbiology 50: 985-987.

de Hoog GS, Dukik K, Monod M, et al. 2017. Toward a novel multilocus phylogenetic taxonomy for the dermatophytes. Mycopathologia 182: 5-31.

Debourgogne A, Gueidan C, de Hoog S, Lozniewski A, Machouart M. 2012. Comparison of two DNA sequence-based typing schemes for the Fusarium solani species complex and proposal of a new consensus method. Journal of Microbiological Methods 91: 65-72.

Dhib I, Khammari I, Yaacoub A, et al. 2017. Relationship between phenotypic and genotypic characteristics of Trichophyton mentagrophytes strains isolated from patients with dermatophytosis. Mycopathologia 182: 487-493. 
Drouot S, Mignon B, Fratti M, Roosje P, Monod M. 2009. Pets as the main source of two zoonotic species of the Trichophyton mentagrophytes complex in Switzerland, Arthroderma vanbreuseghemii and Arthroderma benhamiae. Veterinary Dermatology 20: 13-18.

Duarte A, Castro I, da Fonseca IMP, et al. 2010. Survey of infectious and parasitic diseases in stray cats at the Lisbon Metropolitan Area, Portugal. Journal of Feline Medicine and Surgery 12: $441-446$.

Dukik K, Freeke J, Jamalian A, et al. 2018. Ultra-high-resolution mass spectrometry for identification of closely related dermatophytes with different clinical predilections. Journal of Clinical Microbiology 56: e00102-00118.

Dvořák J, Otčenášek M. 1969. Mycological diagnosis of animal dermatophytoses. Academia, Prague, Czech Republic.

Dvořák J, Otčenášek M, Komárek J. 1965. Das Spektrum der aus Tierläsionen in Ostböhmen in den Jahren 1962-1964 isolierten Dermatophyten. Mycoses 8: 126-127.

Ehrich D. 2006. AFLPdat: a collection of R functions for convenient handling of AFLP data. Molecular Ecology Notes 6: 603-604.

English MP, Evans CD, Hewitt M, Warin RP. 1962. Hedgehog ringworm. British Medical Journal 1: $149-151$.

Evanno G, Regnaut S, Goudet J. 2005. Detecting the number of clusters of individuals using the software STRUCTURE: a simulation study. Molecular Ecology 14: 2611-2620.

Fréalle E, Rodrigue M, Gantois N, et al. 2007. Phylogenetic analysis of Trichophyton mentagrophytes human and animal isolates based on MnSOD and ITS sequence comparison. Microbiology 153: 3466-3477.

Fumeaux J, Mock M, Ninet B, et al. 2004. First report of Arthroderma benhamiae in Switzerland. Dermatology 208: 244-250.

Gardes M, Bruns TD. 1993. ITS primers with enhanced specificity for basidiomycetes-application to the identification of mycorrhizae and rusts. Molecular Ecology 2: 113-118.

Georg LK. 1960. Animal ringworm in public health: diagnosis and nature. US Government Printing Office, Washington, USA.

Glass NL, Donaldson GC. 1995. Development of primer sets designed for use with the PCR to amplify conserved genes from filamentous ascomycetes. Applied and Environmental Microbiology 61: 1323-1330.

Gräser Y, De Hoog S, Summerbell R. 2006. Dermatophytes: recognizing species of clonal fungi. Medical Mycology 44: 199-209.

Gräser Y, Fröhlich J, Presber W, de Hoog S. 2007. Microsatellite markers reveal geographic population differentiation in Trichophyton rubrum. Journal of Medical Microbiology 56: 1058-1065. 
Gräser Y, Kuijpers AFA, Presber W, De Hoog GS. 2000. Molecular taxonomy of the Trichophyton rubrum complex. Journal of Clinical Microbiology 38: 3329-3336.

Gräser Y, Scott J, Summerbell R. 2008. The new species concept in dermatophytes - a polyphasic approach. Mycopathologia 166: 239-256.

Grisólia ME. 2019. Perfil de sensibilidade aos antifúngicos e de variabilidade genética de espécies de Trichophyton isolados de pacientes com infecção cutânea atendidos em um Serviço Público de Micologia em Manaus/AM. Doctoral Thesis, Instituto Leônidas e Maria Deane, Fundação Oswaldo Cruz, Manaus, Brazil.

Guillot J, Decaudin B, Bulliot C, et al. 2018. Emergence of Trichophyton benhamiae in guinea pigs: a retrospective study from the mycology laboratory of the veterinary college of Alfort. Medical Mycology 56: S55-S55.

Hadrich I, Ranque S. 2015. Typing of fungi in an outbreak setting: lessons learned. Current Fungal Infection Reports 9: 314-323.

Hainsworth S, Hubka V, Lawrie AC, et al. 2020. Predominance of Trichophyton interdigitale revealed in podiatric nail dust collections in Eastern Australia. Mycopathologia 185: 175-185.

Havlickova B, Czaika V, Friedrich M. 2008. Epidemiological trends in skin mycoses worldwide. Mycoses 51(Suppl. 4): 2-15.

Hayette M-P, Sacheli R. 2015. Dermatophytosis, trends in epidemiology and diagnostic approach. Current Fungal Infection Reports 9: 164-179.

Hedayati MT, Ansari S, Ahmadi B, et al. 2019. Identification of clinical dermatophyte isolates obtained from Iran by matrix-assisted laser desorption/ionization time-of-flight mass spectrometry. Current Medical Mycology 5: 22-26.

Heidemann S, Monod M, Gräser Y. 2010. Signature polymorphisms in the internal transcribed spacer region relevant for the differentiation of zoophilic and anthropophilic strains of Trichophyton interdigitale and other species of T. mentagrophytes sensu lato. British Journal of Dermatology 162: 282-295.

Hejtmánek M, Hejtmánková N. 1989. Hybridization and sexual stimulation in Trichophyton mentagrophytes. Folia Microbiologica 34: 77-79.

Hiruma J, Kano R, Harada K, et al. 2015. Occurrence of Arthroderma benhamiae genotype in Japan. Mycopathologia 179: 219-223.

Hubka V, Barrs V, Dudová Z, et al. 2018a. Unravelling species boundaries in the Aspergillus viridinutans complex (section Fumigati): opportunistic human and animal pathogens capable of interspecific hybridization. Persoonia 41: 142-174.

Hubka V, Čmoková A, Peano A, et al. 2018b. Zoonotic dermatophytoses: clinical manifestation, diagnosis, etiology, treatment, epidemiological situation in the Czech Republic. Československá Dermatologie 93: 208-235. 
Hubka V, Nissen C, Jensen R, et al. 2015a. Discovery of a sexual stage in Trichophyton onychocola, a presumed geophilic dermatophyte isolated from toenails of patients with a history of $T$. rubrum onychomycosis. Medical Mycology 53: 798-809.

Hubka V, Novakova A, Kolarik M, Jurjevic Z, Peterson SW. 2015b. Revision of Aspergillus section Flavipedes: seven new species and proposal of section Jani sect. nov. Mycologia 107: 169208.

Hubka V, Peano A, Cmokova A, Guillot J. 2018c. Common and emerging dermatophytoses in animals: well-known and new threats. In: Seyedmousavi S, de Hoog GS, Guillot J, Verweij PE (eds), Emerging and Epizootic Fungal Infections in Animals, Springer, Cham, Switzerland: 31-79.

Hubka V, Větrovský T, Dobiášová S, et al. 2014. Molecular epidemiology of dermatophytoses in the Czech Republic - two-year-study results. Česko-slovenská Dermatologie 89: 167-174.

Hunter PR, Gaston MA. 1988. Numerical index of the discriminatory ability of typing systems: an application of Simpson's index of diversity. Journal of Clinical Microbiology 26: 2465-2466.

Huson DH. 1998. SplitsTree: analyzing and visualizing evolutionary data. Bioinformatics 14: 68-73.

Chang Y, Wickes BL, Miller G, Penoyer L, Kwon-Chung K. 2000. Cryptococcus neoformans STE1 $2 \alpha$ regulates virulence but is not essential for mating. The Journal of experimental medicine 191: 871-882.

Charlent A-L. 2011. Le complexe Tricophyton mentagrophytes, caractérisation mycologique et moléculaire d'un nouveau variant: Trichophyton mentagrophytes var. porcellae. $\mathrm{PhD}$ Thesis, Faculté de Pharmacie, Université Henri Poincaré, Nancy, France.

Cheema MS, Christians JK. 2011. Virulence in an insect model differs between mating types in Aspergillus fumigatus. Medical Mycology 49: 202-207.

Chermette R, Ferreiro L, Guillot J. 2008. Dermatophytoses in animals. Mycopathologia 166: 385405.

Choi JS, Gräser Y, Walther G, et al. 2012. Microsporum mirabile and its teleomorph Arthroderma mirabile, a new dermatophyte species in the M. cookei clade. Medical Mycology 50: 161-169.

Jun JB, Sang YH, Chung SL, Choi JS, Suh SB. 2004. The mycological and molecular biological studies on Arthroderma benhamiae isolated for the first time in Korea. Korean Journal of Medical Mycology 9: 12-27.

Kandemir H, Dukik K, Hagen F, et al. 2020. Polyphasic discrimination of Trichophyton tonsurans and T. equinum from humans and horses. Mycopathologia 185: 113-122.

Kane M, Summerbell R. 1997. Laboratory handbook of dermatophytes. A clinical guide and laboratory manual of dermatophytes and other filamentous fungi from skin, hair and nails.

Kano R, Kawasaki M, Mochizuki T, Hiruma M, Hasegawa A. 2012. Mating genes of the Trichophyton mentagrophytes complex. Mycopathologia 173: 103-112. 
Kano R, Nakamura Y, Yasuda K, et al. 1998. The first isolation of Arthroderma benhamiae in Japan. Microbiology and Immunology 42: 575-578.

Kano R, Yoshida E, Yaguchi T, et al. 2014. Mating type gene (MAT1-2) of Trichophyton verrucosum. Mycopathologia 177: 103-112.

Kargl A, Kosse B, Uhrlaß S, et al. 2018. Hedgehog fungi in a dermatological office in Munich: case reports and review. Hautarzt 69: 576-585.

Kaszubiak A, Klein S, De Hoog G, Gräser Y. 2004. Population structure and evolutionary origins of Microsporum canis, M. ferrugineum and M. audouinii. Infection, Genetics and Evolution 4: 179-186.

Katoh K, Rozewicki J, Yamada KD. 2017. MAFFT online service: multiple sequence alignment, interactive sequence choice and visualization. Briefings in Bioinformatics 20: 1160-1166.

Kawasaki M. 2011. Verification of a taxonomy of dermatophytes based on mating results and phylogenetic analyses. Medical Mycology Journal 52: 291-295.

Kawasaki M, Anzawa K, Mochizuki T, Ishizaki H, M. Hemashettar B. 2009. Successful mating of a human isolate of Arthroderma simii with a tester strain of A. vanbreuseghemii. Medical Mycology Journal 50: 15-18.

Kawasaki M, Anzawa K, Ushigami T, Kawanishi J, Mochizuki T. 2011. Multiple gene analyses are necessary to understand accurate phylogenetic relationships among Trichophyton species. Medical Mycology Journal 52: 245-254.

Kawasaki M, Anzawa K, Wakasa A, et al. 2010. Matings among three teleomorphs of Trichophyton mentagrophytes. Japanese Journal of Medical Mycology 51: 143-152.

Kelly KL. 1964. Inter-society color council - National bureau of standards color name charts illustrated with centroid colors. US Government Printing Office, Washington, USA.

Khettar L, Contet-Audonneau N. 2012. Cochon d'Inde et dermatophytose. Annales de Dermatologie et de Venereologie 139: 631-635.

Khosravi A, Mahmoudi M. 2003. Dermatophytes isolated from domestic animals in Iran. Mycoses 46: 222-225.

Kimura U, Yokoyama K, Hiruma M, et al. 2015. Tinea faciei caused by Trichophyton mentagrophytes (molecular type Arthroderma benhamiae) mimics impetigo: a case report and literature review of cases in Japan. Medical Mycology Journal 56: E1-E5.

Kosanke S, Hamann L, Kupsch C, et al. 2018. Unequal distribution of the mating type (MAT) locus idiomorphs in dermatophyte species. Fungal Genetics and Biology 118: 45-53.

Kosman E. 2003. Nei's gene diversity and the index of average differences are identical measures of diversity within populations. Plant Pathology 52: 533-535.

Kraemer A, Hein J, Heusinger A, Mueller R. 2013. Clinical signs, therapy and zoonotic risk of pet guinea pigs with dermatophytosis. Mycoses 56: 168-172. 
Kraemer A, Mueller R, Werckenthin C, Straubinger R, Hein J. 2012. Dermatophytes in pet guinea pigs and rabbits. Veterinary Microbiology 157: 208-213.

Kupsch C, Berlin M, Gräser Y. 2017. Dermophytes and guinea pigs: An underestimated danger? Hautarzt 68: 827-830.

Kupsch C, Berlin M, Ritter L, et al. 2020. The guinea pig fungus Trichophyton benhamiae Germany-wide distribution analysis of the zoonotic agent. In: Groschup MH, Ludwig S, Drosten C (eds), Zoonoses 2019 - International Symposium on Zoonoses Research, Berlin, Germany, Journal der Deutschen Dermatologischen Gesellschaft 18: 12.

Lanfear R, Frandsen PB, Wright AM, Senfeld T, Calcott B. 2017. PartitionFinder 2: new methods for selecting partitioned models of evolution for molecular and morphological phylogenetic analyses. Molecular Biology and Evolution 34: 772-773.

Lebasque J. 1933. Les champignons des teignes du cheval et des bovidés. PhD Thesis, Faculté des Sciences de Paris, Paris, France.

Lee WJ, Eun DH, Jang YH, et al. 2018. Tinea faciei in a mother and daughter caused by Arthroderma benhamiae. Annals of Dermatology 30: 241-242.

Leigh JW, Bryant D. 2015. POPART: full-feature software for haplotype network construction. Methods in Ecology and Evolution 6: 1110-1116.

Lund A, Bratberg AM, Næss B, Gudding R. 2014. Control of bovine ringworm by vaccination in Norway. Veterinary Immunology and Immunopathology 158: 37-45.

Lysková P, Dobiáš R, Kuklová I, et al. 2018. Five cases of dermatophytosis in man caused by zoophilic species Trichophyton erinacei transmitted from hedgehogs. Česko-slovenská Dermatologie 93: 237-243.

Lysková P, Hubka V, Petřičáková A, et al. 2015. Equine dermatophytosis due to Trichophyton bullosum, a poorly known zoophilic dermatophyte masquerading as T. verrucosum. Mycopathologia 180: 407-419.

Maitte C, Leterrier M, Le Pape P, Miegeville M, Morio F. 2013. Multilocus sequence typing of Pneumocystis jirovecii from clinical samples: how many and which loci should be used? Journal of Clinical Microbiology 51: 2843-2849.

Martins WS, Lucas DCS, de Souza Neves KF, Bertioli DJ. 2009. WebSat-A web software for microsatellite marker development. Bioinformation 3: 282-283.

Matute DR, Sepúlveda VE. 2019. Fungal species boundaries in the genomics era. Fungal Genetics and Biology 131: 103249.

Metin B, Heitman J. 2017. Sexual reproduction in dermatophytes. Mycopathologia 182: 45-55.

Meyer W, Aanensen DM, Boekhout T, et al. 2009. Consensus multi-locus sequence typing scheme for Cryptococcus neoformans and Cryptococcus gattii. Medical Mycology 47: 561-570. 
Mirhendi H, Makimura K, de Hoog GS, et al. 2015. Translation elongation factor 1- $\alpha$ gene as a potential taxonomic and identification marker in dermatophytes. Medical Mycology 53: 215224.

Mochizuki T, Kawasaki M, Ishizaki H, et al. 2001. Molecular epidemiology of Arthroderma benhamiae, an emerging pathogen of dermatophytoses in Japan, by polymorphisms of the non-transcribed spacer region of the ribosomal DNA. Journal of Dermatological Science 27: 14-20.

Mochizuki T, Takeda K, Anzawa K. 2017. Molecular markers useful for intraspecies subtyping and strain differentiation of dermatophytes. Mycopathologia 182: 57-65.

Mochizuki T, Watanabe S, Kawasaki M, Tanabe H, Ishizaki H. 2002. A Japanese case of tinea corporis caused by Arthroderma benhamiae. The Journal of dermatology 29: 221-225.

Moretti A, Agnetti F, Mancianti F, et al. 2013. Dermatophytosis in animals: epidemiological, clinical and zoonotic aspects. Giornale Italiano di Dermatologia e Venereologia 148: 563-572.

Morris P, English MP. 1969. Trichophyton mentagrophytes var. erinacei in British hedgehogs. Sabouraudia 7: 122-128.

Morris P, English MP. 1973. Transmission and course of Trichophyton erinacei infections in British hedgehogs. Sabouraudia 11: 42-47.

Müller K. 2005. SeqState. Applied Bioinformatics 4: 65-69.

Needle DB, Gibson R, Hollingshead NA, et al. 2019. Atypical Dermatophytosis in 12 North American Porcupines (Erethizon dorsatum) from the Northeastern United States 2010-2017. Pathogens 8: 171.

Nei M. 1987. Molecular evolutionary genetics. Columbia University Press, New York, USA.

Nenoff P, Erhard M, Simon JC, et al. 2013. MALDI-TOF mass spectrometry-a rapid method for the identification of dermatophyte species. Medical Mycology 51: 17-24.

Nenoff P, Uhrlaß S, Krüger C, et al. 2014. Trichophyton species von Arthroderma benhamiae - a new infectious agent in dermatology. Journal der Deutschen Dermatologischen Gesellschaft 12: 571-582.

Nenoff P, Verma SB, Uhrlaß S, Burmester A, Gräser Y. 2019. A clarion call for preventing taxonomical errors of dermatophytes using the example of the novel Trichophyton mentagrophytes genotype VIII uniformly isolated in the Indian epidemic of superficial dermatophytosis. Mycoses 62: 6-10.

Nguyen L-T, Schmidt HA, von Haeseler A, Minh BQ. 2015. IQ-TREE: A fast and effective stochastic algorithm for estimating maximum-likelihood phylogenies. Molecular Biology and Evolution 32: $268-274$.

Overgaauw P, van Avermaete K, Mertens C, Meijer M, Schoemaker N. 2017. Prevalence and zoonotic risks of Trichophyton mentagrophytes and Cheyletiella spp. in guinea pigs and rabbits in Dutch pet shops. Veterinary Microbiology 205: 106-109. 
Packeu A, Stubbe D, Roesems S, et al. 2020. Lineages within the Trichophyton rubrum complex. Mycopathologia 185: 123-136.

Padhye A, Carmichael J. 1972. Arthroderma insingulare sp. nov., another gymnoascaceous state of the Trichophyton terrestre complex. Sabouraudia 10: 47-51.

Papegaay J. 1925. Over pathogene huidschimmels in Amsterdam voorkomend bij den mensch. Nederlands Tijdschrift voor Geneeskunde 69: 879-890.

Parker Jr ED. 1979. Ecological implications of clonal diversity in parthenogenetic morphospecies. American Zoologist 19: 753-762.

Pasquetti M, Peano A, Soglia D, et al. 2013. Development and validation of a microsatellite markerbased method for tracing infections by Microsporum canis. Journal of Dermatological Science 70: 123-129.

Pchelin IM, Azarov DV, Churina MA, et al. 2019. Species boundaries in the Trichophyton mentagrophytes/T. interdigitale species complex. Medical Mycology 57: 781-789.

Pchelin IM, Zlatogursky VV, Rudneva MV, et al. 2016. Reconstruction of phylogenetic relationships in dermatomycete genus Trichophyton Malmsten 1848 based on ribosomal internal transcribed spacer region, partial 28S rRNA and beta-tubulin genes sequences. Mycoses 59: 566-575.

Piérard-Franchimont C, Hermanns J-F, Collette C, Pierard G, Quatresooz P. 2008. Hedgehog ringworm in humans and a dog. Acta Clinica Belgica 63: 322-324.

Pihet M, Bourgeois H, Mazière J-Y, et al. 2008. Isolation of Trichophyton concentricum from chronic cutaneous lesions in patients from the Solomon Islands. Transactions of the Royal Society of Tropical Medicine and Hygiene 102: 389-393.

Prakash A, Sharma C, Singh A, et al. 2016. Evidence of genotypic diversity among Candida auris isolates by multilocus sequence typing, matrix-assisted laser desorption ionization time-offlight mass spectrometry and amplified fragment length polymorphism. Clinical Microbiology and Infection 22: 277.e271-277.e279.

Pritchard JK, Stephens M, Donnelly P. 2000. Inference of population structure using multilocus genotype data. Genetics 155: 945-959.

R_Core_Team. 2016. R: A language and environment for statistical computing. R Foundation for Statistical Computing, Vienna, Austria.

Ranjbar R, Karami A, Farshad S, Giammanco GM, Mammina C. 2014. Typing methods used in the molecular epidemiology of microbial pathogens: a how-to guide. New Microbiologica 37: 115.

Réblová M, Hubka V, Thureborn O, et al. 2016. From the tunnels into the treetops: new lineages of black yeasts from biofilm in the Stockholm metro system and their relatives among antassociated fungi in the Chaetothyriales. PLoS One 11: e0163396. 
Rezaei-Matehkolaei A, Makimura K, de Hoog S, et al. 2013. Molecular epidemiology of dermatophytosis in Tehran, Iran, a clinical and microbial survey. Medical Mycology 51: 203207.

Rezaei-Matehkolaei A, Rafiei A, Makimura K, et al. 2016. Epidemiological aspects of dermatophytosis in Khuzestan, southwestern Iran, an update. Mycopathologia 181: 547-553.

Rippon JW. 1988. Medical Mycology. The pathogenic fungi and the pathogenic actinomycetes. Saunders, Philadelphia, USA.

Ronquist F, Teslenko M, van der Mark P, et al. 2012. MrBayes 3.2: efficient Bayesian phylogenetic inference and model choice across a large model space. Systematic Biology 61: 539-542.

Sabou M, Denis J, Boulanger N, et al. 2018. Molecular identification of Trichophyton benhamiae in Strasbourg, France: a 9-year retrospective study. Medical Mycology 56: 723-734.

Santana AE, Reche-Junior A, Sellera FP, Taborda CP. 2020. A comment on "First report of tinea corporis caused by Arthroderma benhamiae in Brazil". Brazilian Journal of Microbiology: doi:10.1007/s42770-42019-00220-42770.

Seebacher C, Bouchara J-P, Mignon B. 2008. Updates on the epidemiology of dermatophyte infections. Mycopathologia 166: 335-352.

Sharma R, De Hoog S, Presber W, Gräser Y. 2007. A virulent genotype of Microsporum canis is responsible for the majority of human infections. Journal of Medical Microbiology 56: 13771385.

Sharma R, Presber W, Rajak RC, Gräser Y. 2008. Molecular detection of Microsporum persicolor in soil suggesting widespread dispersal in central India. Medical Mycology 46: 67-73.

Shenoy MM, Jayaraman J. 2019. Epidemic of difficult-to-treat tinea in India: Current scenario, culprits, and curbing strategies. Archives of Medicine and Health Sciences 7: 112-117.

Schauder S, Kirsch-Nietzki M, Wegener S, Switzer E, Qadripur S. 2007. Von Igeln auf Menschen: Zoophile Dermatomykose durch Trichophyton erinacei bei 8 Patienten. Hautarzt 58: 62-67.

Schlueter PM, Harris SA. 2006. Analysis of multilocus fingerprinting data sets containing missing data. Molecular Ecology Notes 6: 569-572.

Schneider S, Roessli D, Excoffier L. 2000. ARLEQUIN: a software for population genetics data analysis, Version 2.000. University of Geneva, Geneva, Switzerland.

Schönswetter P, Tribsch A. 2005. Vicariance and dispersal in the alpine perennial Bupleurum stellatum L. (Apiaceae). Taxon 54: 725-732.

Schrödl W, Heydel T, Schwartze VU, et al. 2012. Direct analysis and identification of pathogenic Lichtheimia species by matrix-assisted laser desorption ionization-time of flight analyzermediated mass spectrometry. Journal of Clinical Microbiology 50: 419-427.

Schuelke M. 2000. An economic method for the fluorescent labeling of PCR fragments. Nature Biotechnology 18: 233-234. 
Sieklucki U, Oh SH, Hoyer LL. 2014. Frequent isolation of Arthroderma benhamiae from dogs with dermatophytosis. Veterinary Dermatology 25: 39-41.

Silver S, Vinh DC, Embil JM. 2008. The man who got too close to his cows. Diagnostic Microbiology and Infectious Disease 60: 419-420.

Singh A, Masih A, Monroy-Nieto J, et al. 2019. A unique multidrug-resistant clonal Trichophyton population distinct from Trichophyton mentagrophytes/Trichophyton interdigitale complex causing an ongoing alarming dermatophytosis outbreak in India: Genomic insights and resistance profile. Fungal Genetics and Biology 133: 103266.

Sitterle E, Frealle E, Foulet F, et al. 2012. Trichophyton bullosum: a new zoonotic dermatophyte species. Medical Mycology 50: 305-309.

Skořepová M, Hubka V, Polášková S, Stará J, Čmoková A. 2014. Our first experiences with Infections caused by Arthroderma benhamiae (Trichophyton sp.). Česko-slovenská Dermatologie 89: 192-198.

Smith J, Marples MJ. 1964. Trichophyton mentagrophytes var. erinacei. Sabouraudia 3: 1-10. Steenkamp ET, Wingfield MJ, McTaggart AR, Wingfield BD. 2018. Fungal species and their boundaries matter - Definitions, mechanisms and practical implications. Fungal Biology Reviews 32: 104-116.

Stockdale PM. 1964. The Microsporum gypseum complex (Nannizzia incurvata Stockd., N. gypsea (Nann.) comb. nov., N. fulva sp. nov.). Sabouraudia 3: 114-126.

Su H, Packeu A, Ahmed SA, et al. 2019. Species distinction in the Trichophyton rubrum complex. Journal of Clinical Microbiology 57: e00352-00319.

Suh S-O, Grosso KM, Carrion ME. 2018. Multilocus phylogeny of the Trichophyton mentagrophytes species complex and the application of matrix-assisted laser desorption/ionization-time-offlight (MALDI-TOF) mass spectrometry for the rapid identification of dermatophytes. Mycologia 110: 118-130.

Summerbell R. 2002. What is the evolutionary and taxonomic status of asexual lineages in the dermatophytes? Studies in Mycology 47: 97-101.

Summerbell RC. 2011. Trichophyton, Microsporum, Epidermophyton, and agents of superficial mycoses. In: Versalovic J, Carroll K, Funke G, et al. (eds), Manual of Clinical Microbiology, 10th Edition, American Society of Microbiology, Washington: 1919-1942.

Summerbell RC, Moore MK, Starink-Willemse M, Van Iperen A. 2007. ITS barcodes for Trichophyton tonsurans and T. equinum. Medical Mycology 45: 193-200.

Symoens F, Jousson O, Packeu A, et al. 2013. The dermatophyte species Arthroderma benhamiae: intraspecies variability and mating behaviour. Journal of Medical Microbiology 62: 377-385.

Taghipour S, Pchelin IM, Zarei Mahmoudabadi A, et al. 2019. Trichophyton mentagrophytes and T. interdigitale genotypes are associated with particular geographic areas and clinical manifestations. Mycoses 62: 1084-1091. 
Takahashi H, Takahashi-Kyuhachi H, Takahashi Y, et al. 2008. An intrafamilial transmission of Arthroderma benhamiae in Canadian porcupines (Erethizon dorsatum) in a Japanese zoo. Medical Mycology 46: 465-473.

Takahashi Y, Haritani K, Sano A, et al. 2002. An isolate of Arthroderma benhamiae with Trichophyton mentagrophytes var. erinacei anamorph isolated from a four-toed hedgehog (Atelerix albiventris) in Japan. Japanese Journal of Medical Mycology 43: 249-255.

Takahashi Y, Sano A, Takizawa K, et al. 2003. The epidemiology and mating behavior of Arthroderma benhamiae var. erinacei in household four-toed hedgehogs (Atelerix albiventris) in Japan. Japanese Journal of Medical Mycology 44: 31-38.

Takashio M. 1974. Observations on African and European strains of Arthroderma benhamiae. International Journal of Dermatology 13: 94-101.

Takeda K, Nishibu A, Anzawa K, Mochizuki T. 2012. Molecular epidemiology of a major subgroup of Arthroderma benhamiae isolated in Japan by restriction fragment length polymorphism analysis of the non-transcribed spacer region of ribosomal RNA gene. Japanese Journal of Infectious Diseases 65: 233-239.

Tan J, Liu X, Gao Z, et al. 2020. A case of Tinea Faciei caused by Trichophyton benhamiae: first report in China. BMC Infectious Diseases 20: 1-5.

Tartor YH, El Damaty HM, Mahmmod YS. 2016. Diagnostic performance of molecular and conventional methods for identification of dermatophyte species from clinically infected Arabian horses in Egypt. Veterinary Dermatology 27: 401-e102.

Taylor JW, Hann-Soden C, Branco S, Sylvain I, Ellison CE. 2015. Clonal reproduction in fungi. Proceedings of the National Academy of Sciences 112: 8901-8908.

Turland NJ, Wiersema JH, Barrie FR, et al. 2018. International Code of Nomenclature for algae, fungi, and plants (Shenzhen Code) adopted by the Nineteenth International Botanical Congress Shenzhen, China, July 2017. Koeltz Botanical Books, Glashütten, Germany.

Uhrlaß S, Krüger C, Nenoff P. 2015. Microsporum canis: Aktuelle Daten zur Prävalenz des zoophilen Dermatophyten im mitteldeutschen Raum. Der Hautarzt 66: 855-862.

Uhrlaß S, Schroedl W, Mehlhorn C, et al. 2018. Molecular epidemiology of Trichophyton quinckeanum-a zoophilic dermatophyte on the rise. Journal der Deutschen Dermatologischen Gesellschaft 16: 21-32.

Vu D, Groenewald M, De Vries M, et al. 2019. Large-scale generation and analysis of filamentous fungal DNA barcodes boosts coverage for kingdom fungi and reveals thresholds for fungal species and higher taxon delimitation. Studies in Mycology 92: 135-154.

White TJ, Bruns T, Lee S, Taylor J. 1990. Amplification and direct sequencing of fungal ribosomal RNA genes for phylogenetics. In: Innis MA, Gelfand DH, J. SJ, White TJ (eds), PCR protocols: a guide to methods and applications, Academic Press, San Diego, USA: 315-322. 
1 Woodgyer A. 2004. The curious adventures of Trichophyton equinum in the realm of molecular

2

3

4

5

6

7

8

9

10

11

12

13

14

13 biology: a modern fairy tale. Medical Mycology 42: 397-403.

Yeh FC, Boyle T, Rongcai Y, et al. 1999. POPGENE version 1.31. A Microsoft window based freeware for population genetic analysis. University of Alberta, Canada.

Yue C, Cavallo LM, Alspaugh JA, et al. 1999. The STE12 $\alpha$ homolog is required for haploid filamentation but largely dispensable for mating and virulence in Cryptococcus neoformans. Genetics 153: 1601-1615.

Zhan P, Dukik K, Li D, et al. 2018. Phylogeny of dermatophytes with genomic character evaluation of clinically distinct Trichophyton rubrum and T. violaceum. Studies in Mycology 89: 153-175.

Ziółkowska G, Nowakiewicz A, Gnat S, et al. 2015. Molecular identification and classification of Trichophyton mentagrophytes complex strains isolated from humans and selected animal species. Mycoses 58: 119-126. 
2 Figure 1. Chronology of reports of Trichophyton benhamiae phenotypes from various countries. 3 Yellow-phenotype isolates correspond to T. benhamiae var. luteum proposed in this study. White4 phenotype strains correspond to $T$. benhamiae var. benhamiae and two novel species proposed here: $T$. europaeum and $T$. japonicum. The reports are mostly sorted according to the phenotypic characters of cultures reported by the authors and, in more recent studies, by a combination of DNA sequencing and morphology. The icons of the hosts are explained in Figure S1.

Figure 2. Multilocus phylogeny of the Trichophyton benhamiae complex inferred with the maximum likelihood method based on the gapdh, tubb, ITS rDNA and tefl- $\alpha$ loci (alignment characteristics, partitioning scheme and substitution models are listed in Tab S3). Maximum likelihood bootstrap values and Bayesian posterior probabilities are appended to the nodes; only support values higher than $70 \%$ and 0.90 , respectively, are shown. The ex-type strains are designated with a superscripted T. Trichophyton rubrum CBS 202.88 was used as the outgroup.

Figure 3. Haplotype network of the Trichophyton benhamiae clade based on multilocus data (gapdh, tubb, ITS rDNA and tefl- $\alpha$ loci). Haplotypes are indicated by circles whose sizes correspond to the number of analysed strains, and dashes on the connecting lines indicate substitutions (indels are excluded). The upper figure shows the species identity and genotypic diversity, the middle figure shows the distribution of MAT gene idiomorphs, and the lower figure shows the geographic distribution of particular genotypes.

Figure 4. Plot of mean genotypic diversity as a function of the number of microsatellite loci.

Figure 5. The population structure of the Trichophyton benhamiae clade (ten microsatellite loci, 318 isolates). The neighbour-joining tree was calculated from the multilocus microsatellite profiles using the Jaccard distance matrix measure in FAMD 1.3 (Schlueter Harris 2006) and is used solely for the comprehensive presentation of the results. Genetic structure was revealed with STRUCTURE software by Bayesian clustering (the peak of $\Delta \mathrm{K}$ was observed at $\mathrm{K}=6$ ); clones were discarded from the analysis; the number of isolates representing each haplotype is indicated in parentheses following the isolate number; the geographic origin of the isolates representing particular haplotypes is indicated using abbreviations: Europe (Eu), Japan (Jpn), United States of America (USA). Individual haplotypes are represented by horizontal bars; the colours were attributed according to the clusters delimited by STRUCTURE. 
1 Figure 6. Population structure of the Trichophyton benhamiae clade revealed by the analysis of ten microsatellite loci in 318 strains. The NeighborNet network was built with FAMD 1.3 software and visualized in SplitsTree 4.13 using the Jaccard index-based distance matrix (Delta score: 0.1778, Qresidual score: 0.01222). The assignment of strains to main clusters and species is indicated by different colours. The labels of each cluster show the geographic origin of strains with the number of isolates and main host(s). The icons of the hosts are explained in Figure S1.

Figure 7. Phylogenetic tree of the Trichophyton benhamiae clade revealed by the analysis of ten microsatellite loci in 318 strains constructed in FAMD software using a Jaccard index-based distance matrix. Coloured circles display the genotype diversity of the ITS, gapdh and tefl- $\alpha$ loci and the distribution of MAT gene idiomorphs (blue: MAT1-1-1; pink: MAT1-2-1) across Trichophyton benhamiae clade species. Isolate numbers are displayed in Figure S6.

Figure 8. Histograms showing the frequency of pairwise genetic differences within the population of Trichophyton benhamiae var. benhamiae clusters C2 and C3 (A); Trichophyton benhamiae var. luteum (B); Trichophyton japonicum (C); Trichophyton europaeum (D).

Figure 9. Histogram of the simulated index of association $\left(\mathrm{I}_{\mathrm{A}}\right)$ from 10000 permutations of randomization tests under a null model of allelic recombination; the observed values of $\mathrm{I}_{\mathrm{A}}$ are indicated with an arrow.

Figure 10. Overview of the macromorphology of the Trichophyton benhamiae complex taxa on three media (SAB, MEA and PDA) cultivated for 14 days at $25^{\circ} \mathrm{C}$.

Figure 11. Growth rates of Trichophyton benhamiae complex members on three media (SAB, MEA and PDA) and at three different temperatures $\left(25,30\right.$ and $37{ }^{\circ} \mathrm{C}$, on SAB only) after 7 days of cultivation; circles represent median values and the whiskers span the minimum and maximum values.

Figure 12. Length and width of microconidia in taxa belonging to the Trichophyton benhamiae complex. The horizontal lines indicate mean value and interquartile range, whiskers span the $5 \%$ and 95\% percentiles and circles extreme outliers.

Figure 13. Principal component analysis (PCA) of morphological characteristics. The two major axes of the plot show all variables, including the growth rates (cultivation on MEA, SAB, and PDA at 25, 30 and $37^{\circ} \mathrm{C}$ ) and microconidium sizes (mean values of length and width) (A). The correlation matrix shows the Pearson correlation coefficients between variables such as growth rates (three different media and temperatures) and microconidia sizes (length and width). A darker colour indicates stronger 
correlations, which means that all variables within a growth rate or microconidium size group were strongly correlated (B), indicating the possibility of reducing the number of variables.

Figure 14. MALDI-TOF mass spectra in the Trichophyton benhamiae clade members; only variable regions are shown. Comparison of spectra in the species of the former Americano-European race ( $T$. benhamiae var. benhamiae and T. benhamiae var. luteum, T. europaeum and T. japonicum) and the African race (T. africanum) (A). Comparison of T. europaeum, T. japonicum and T. concentricum (B). Comparison of spectra of T. concentricum and two varieties of T. benhamiae (C-D).

Figure 15. Macromorphology and micromorphology of Trichophyton benhamiae var. benhamiae. Colonies after two weeks of cultivation at $25^{\circ} \mathrm{C}$ on Sabouraud's dextrose agar (A, B), Malt extract agar (C, D) and Potato dextrose agar (E, F). Conidiophores bearing microconidia (G-I) and macroconidia (J); macroconidia (K-P), frequently with mycelial fragments at one or both ends (K-N, $\mathrm{P})$; microconidia (R); spiral hyphae (S). Scale bars $=20 \mu \mathrm{m}$.

Figure 16. Macromorphology and micromorphology of Trichophyton benhamiae var. luteum. Colonies after two weeks of cultivation at $25^{\circ} \mathrm{C}$ on Sabouraud's dextrose agar (A, B), Malt extract agar (C, D) and Potato dextrose agar (E, F). Conidiophores bearing microconidia (G-L); microconidia (M). Scale bars $=20 \mu \mathrm{m}$.

Figure 17. Clinical presentation of infections caused by Trichophyton benhamiae clade species in guinea pigs and humans. Guinea pigs: area of alopecia with scaling located in the temporal area (A) and on the back of guinea pig (B); areas with scaling on the ear (C); itchy area of alopecia behind the ear (D); weeping lesion under the eye (E). Zoonotic infections in humans: tinea corporis located on the thigh $(\mathrm{F})$ and chest $(\mathrm{G})$, tinea faciei $(\mathrm{H})$, tinea barbae $(\mathrm{I})$, tinea capitis profunda $(\mathrm{J}, \mathrm{K})$.

Figure 18. Macromorphology and micromorphology of Trichophyton concentricum. Colonies after three weeks of cultivation at $25^{\circ} \mathrm{C}$ on Sabouraud's dextrose agar (A, B), Malt extract agar (C, D) and Potato dextrose agar (E, F). Vegetative hyphae (G-L), frequently consisting of inflated cells and containing intercalar or terminal chlamydospores $(\mathrm{H}, \mathrm{I})$, occasionally proliferating in a zigzag pattern $(\mathrm{K}, \mathrm{L})$. Scale bars $=20 \mu \mathrm{m}$.

Figure 19. Macromorphology and micromorphology of Trichophyton europaeum. Colonies after two weeks of cultivation at $25^{\circ} \mathrm{C}$ on Sabouraud's dextrose agar (A, B), Malt extract agar (C, D) and Potato dextrose agar (E, F). Conidiophores bearing microconidia (G-I); macroconidia (J-M); microconidia (N); spiral hyphae (O, P). Scale bars $=20 \mu \mathrm{m}$. 
2 Figure 20. Macromorphology and micromorphology of Trichophyton japonicum. Colonies after two weeks of cultivation at $25^{\circ} \mathrm{C}$ on Sabouraud's dextrose agar (A, B), Malt extract agar (C, D) and Potato dextrose agar (E, F). Conidiophores bearing microconidia (G-K); macroconidia (L-P); microconidia $(\mathrm{R})$; spiral hyphae $(\mathrm{S}, \mathrm{T})$. Scale bars $=20 \mu \mathrm{m}$.

Figure 21. Macromorphology and micromorphology of Trichophyton erinacei. Colonies after two weeks of cultivation at $25^{\circ} \mathrm{C}$ on Sabouraud's dextrose agar (A, B), Malt extract agar (C, D) and Potato dextrose agar (E, F). Conidiophores bearing microconidia (G-J) and macroconidia (intermediate forms) (K); macroconidia (L-N); free microconidia and macroconidia (two-celled intermediate forms) $(\mathrm{N})$; intercalary conidia - marked with arrows $(\mathrm{P})$. Scale bars $=20 \mu \mathrm{m}$.

Figure 22. Clinical presentation of infections caused by Trichophyton erinacei clade species in animals and humans. Trichophyton erinacei: four-toed hedgehog (Atelerix albiventris) without apparent clinical signs of infection (A), a source of tinea corporis infection in a pet breeder (Lysková et al. 2018); tinea faciei (B) and tinea corporis on the left forearm (C). Trichophyton verrucosum: discrete, scaling patches of hair loss located on the head and neck of cattle (D, E) and goat (F); tinea corporis on the forearm $(\mathrm{G})$, infection that affected scalp skin after previous injury - the situation after surgical removal of necrotic parts $(\mathrm{H})$.

Figure 23. Macromorphology and micromorphology of Trichophyton eriotrephon. Colonies after two weeks of cultivation at $25^{\circ} \mathrm{C}$ on Sabouraud's dextrose agar (A, B), Malt extract agar (C, D) and Potato dextrose agar (E, F). Conidiophores bearing microconidia and intercalary conidia (G-K); microconidia and intercalary conidia with variable shape (L). Scale bars $=20 \mu \mathrm{m}$.

Figure 24. Macromorphology and micromorphology of Trichophyton verrucosum. Colonies after three weeks of cultivation at $25^{\circ} \mathrm{C}$ on Sabouraud's dextrose agar (A, B), Malt extract agar (C, D) and Potato dextrose agar (E, F). Clumps of vegetative hyphae $(\mathrm{G})$; chlamydospores in chains (H, I); macroconidia (J-M); conidiophores (fertile hyphae) with sessile microconidia (N, O); microconidia (P). Scale bars $=20 \mu \mathrm{m}$.

Figure 25. Macromorphology and micromorphology of Trichophyton africanum. Colonies after two weeks of cultivation at $25^{\circ} \mathrm{C}$ on Sabouraud's dextrose agar (A, B), Malt extract agar (C, D) and Potato dextrose agar (E, F). Conidiophores bearing microconidia (G-K); macroconidia (L-N); microconidia (O). Scale bars $=20 \mu \mathrm{m}$. 
1 Figure 26. Macromorphology and micromorphology of Trichophyton bullosum. Colonies after three

2 weeks of cultivation at $25{ }^{\circ} \mathrm{C}$ on Sabouraud's dextrose agar (A, B), Malt extract agar (C, D) and

3 Potato dextrose agar (E, F). Chladospores in chains and free chlamydospores (G); thick-walled

4 vegetative hyphae with numerous intercalar or terminal chlamydospores (H, I); detail of colony with

5 submerged, dendritic growth on Sabouraud's dextrose agar supplemented with cycloheximide and

6 chloramphenicol after 3 months of cultivation at $25^{\circ} \mathrm{C}(\mathrm{L})$; vegetative hyphae with terminal

7 chlamydospores (M, N). Scale bars $=20 \mu \mathrm{m}$.

8

9

10

Figure 27. Clinical presentation of infections caused by Trichophyton bullosum: patches of hair loss in the saddle area, shoulders, hip bones, withers and upper chest of a horse (A), isolate CCF 4831 (Lysková et al. 2015); scaling patches of hair loss located on the head, chest and legs of a donkey (BE), isolate CCF 5730 .

Figure 28. Overview of selected data on ecology, phenotype and population genetics plotted on the simplified four-gene phylogeny of the Trichophyton benhamiae species complex. The icons of the hosts are explained in Figure S1.

Figure 29. Geographic distribution of species belonging to the Trichophyton benhamiae clade based on ITS rDNA available in GenBank database (Table S10). The main primary host(s) of species in different continents are marked by icons (explained in Figure S1).

\section{LEGENDS TO SUPPLEMENTARY FIGURES}

Figure S1. Legend for the host icons used in this study.

Figure S2. Maximum likelihood tree based on ITS region sequences. Maximum likelihood bootstrap values are appended to the nodes; only support values higher than $70 \%$ are shown; the ex-type strains are designated with a superscripted T; Trichophyton rubrum CBS 202.88 was used as the outgroup. Clades with $>5$ identical sequences are collapsed; positions refer to the alignment available in the Dryad digital repository.

Figure S3. Maximum likelihood tree based on gapdh gene sequences. Maximum likelihood bootstrap values are appended to the nodes; only support values higher than $70 \%$ are shown; the ex-type strains are designated with a superscripted T; Trichophyton rubrum CBS 202.88 was used as the outgroup. Clades with $>5$ identical sequences are collapsed; positions refer to the alignment available in the Dryad digital repository. 
1 Figure S4. Maximum likelihood tree based on tefl- $\alpha$ gene sequences. Maximum likelihood bootstrap 2 values are appended to the nodes; only support values higher than $70 \%$ are shown; the ex-type strains 3 are designated with a superscripted T; Trichophyton rubrum CBS 202.88 was used as the outgroup. 4 Clades with $>5$ identical sequences are collapsed; positions refer to the alignment available in the Dryad 5 digital repository.

6

7 Figure S5. Maximum likelihood tree based on $t u b b$ gene sequences. Maximum likelihood bootstrap values are appended to the nodes; only support values higher than $70 \%$ are shown; the ex-type strains are designated with a superscripted T; Trichophyton rubrum CBS 202.88 was used as the outgroup.

10 Clades with $>5$ identical sequences are collapsed.

Figure S6. Phylogenetic tree of the Trichophyton benhamiae clade revealed by the analysis of ten microsatellite loci in 318 strains constructed in FAMD software using a Jaccard index-based distance matrix. Coloured circles display the genotype diversity of the ITS, gapdh and tefl- $\alpha$ loci and the distribution of MAT gene idiomorphs across Trichophyton benhamiae clade species. 